\title{
Synchronization and Stability of Two Unbalanced Rotors with Fast Antirotation considering Energy Balance
}

\author{
Yongjun Hou and Pan Fang \\ School of Mechanical Engineering, Southwest Petroleum University, Chengdu 610500, China \\ Correspondence should be addressed to Pan Fang; ckfangpan@126.com
}

Received 26 March 2015; Accepted 27 July 2015

Academic Editor: Michael J. Brennan

Copyright ( 2015 Y. Hou and P. Fang. This is an open access article distributed under the Creative Commons Attribution License, which permits unrestricted use, distribution, and reproduction in any medium, provided the original work is properly cited.

\begin{abstract}
We consider synchronization and stability of two unbalanced rotors reversely and fast excited by induction motors fixed on an oscillating body. We explore the energy balance of the system and show how the energy is transferred between the rotors via the oscillating body allowing the implementation of the synchronization of the two rotors. An approximate analytical analysis, energy balance method, allows deriving the synchronization condition, and the stability criterion of the synchronization is deduce by disturbance differential equations. Later, to prove the correctness of the theoretical analysis, many features of the vibrating system are computed and discussed by computer simulations. The proposed method may be useful for analyzing and understanding the mechanism of synchronization, stability, and energy balance of similar fast rotation rotors excited by induction motors in vibrating systems.
\end{abstract}

\section{Introduction}

Synchronization is commonly observed to occur in nature, such as synchronous oscillations of the nuclei and cells of malignant tumors in biological objects and geosynchronous satellites rotating around the earth in celestial mechanics. All synchronous regimes arise due to natural properties of the process themselves and their natural interaction. The history of synchronization investigation goes back to the 17th century when Huygens observed weak synchronization of two pendulum clocks in a ship [1], and his work has attracted attention of many scientists in other subjects. For example, synchronization in acoustic and electroacoustic systems was discovered by Rayleigh [2]. At the 19th century, van der Pol found synchronization of a certain electricalmechanical system [3]. They called synchronization as "frequency capture" or "frequency synchronization." A wellknown example is frequency synchronization of oscillating and rotating bodies in mechanical system, which is also called self-synchronization. Many research methods to the rotorsynchronization have been proposed by many scholars. In the 1960s, Blekhman firstly studied the synchronization and stability theory of mechanical rotors with nonlinear theory and wrote some classical monograph [4]. In the 1980s, Wen proposed average method to explore the theory of synchronization and stability of rotors and successfully applied it to vibration engineering [5]. Recently, combining nonlinear theory with average method, Zhang et al. developed the theory of synchronization and stability of unbalanced rotor systems in the light of the variable parameter of angular velocity of induction motors, and they employ the method and some experiments to investigate the rotor-synchronization and the synchronous stability in diversity vibrating systems $[6,7]$. Fang et al. also used it to investigate the selfsynchronization of two homodromy rotors coupled with a pendulum rod in a far-resonant vibrating system [8]. Sperling et al. presented analytical and numerical investigations of a two-plane automatic balancing device for equilibration of rigid-rotor unbalance [9]. In addition, Balthazar et al. [10, 11] dealt with self-synchronization of two and four nonideal exciters with numerical simulations. These studies to the synchronization and the stability related to vibrating systems greatly facilitate the development of a number of vibratory ore processing machines and lead to many inventions or patents including the self-synchronous vibrating feeders, selfsynchronous vibrating conveyors, self-synchronous vibrating 


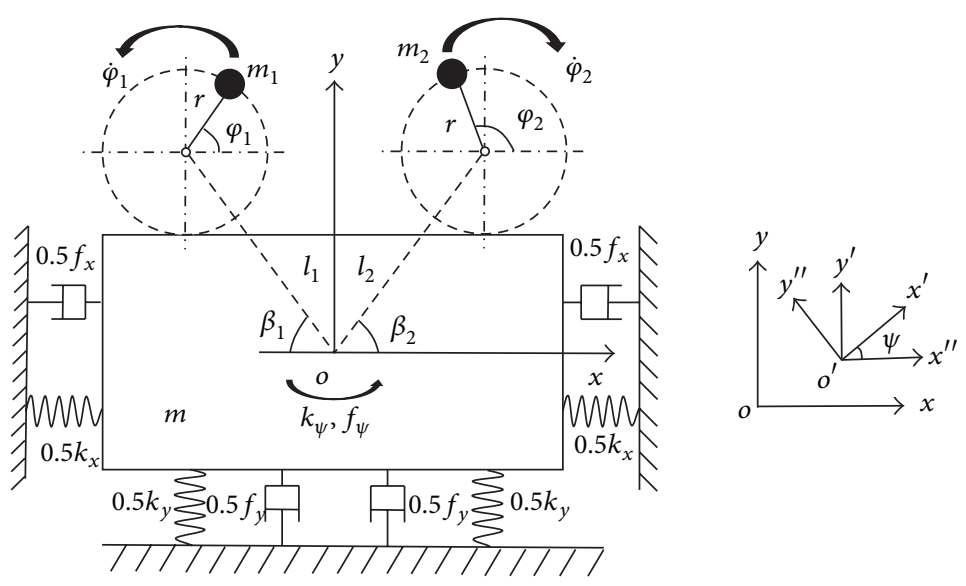

FIGURE 1: Dynamic model of the vibration system.

screens, and synchronous rolling mills. The aforementioned investigation is mainly for the rotor-synchronization or exciter-synchronization in vibrating system, and the phenomenon of synchronization of pendulums hanging on a common moveable beam is another research subject by a number of authors. By the Poincare method and the small parameter method, Jovanovic and Koshkin have studied synchronization and stability of Huygens' clocks [12]. Recently, Koluda and Perlikowski derived the synchronization conditions and explained the energy transmission between double pendula via an oscillating beam with energy balance method [13-15]. Kapitaniak et al. explored synchronous states of slowly rotating pendula considering the oscillating beam moving in a single DOF $[16,17]$. Pena-Ramírez concerned the synchronized motion of Huygens' model building on the original work of Blekhman [18, 19]. Yurchenko et al. described synchronization of rotating parametric pendulums combining stochastic calculus [20]. The works of these authors have been facilitating the significant development in synchronization of pendula.

In this paper, we further extend investigation of a generalization of the vibrating system on synchronization and stability of unbalanced rotors fast excited by induction motors and show how the energy is transferred between rotors via the oscillating body allowing the implementation of synchronization of rotors. We use the type model where two rotors reversely and fast excited by the induction motors are fixed on an oscillating body in a far-resonant vibrating system (i.e., the operating frequency of the system is about 4-10 times of its natural frequency, and the damping value is very small). The performed approximate analytical analysis allows deriving the synchronization condition and stability criterion, in addition to explaining the synchronization discipline with considering diversity features of the vibrating system. Finally, some numerical simulations are performed to prove the correctness of the theoretical analysis.

This paper is organized as follows. Section 2 describes the dynamic model of the proposed vibrating system. In Section 3 we deduce the energy balance of the synchronous state (or steady state) of the system. The synchronization condition and the stability criterion of the two rotors have been identified in Section 4. Section 5 presents the results of our numerical simulations and describes the energy balance of the synchronous states of the system. Finally, we summarize our results in Section 6.

\section{The Dynamic Model of System}

The analyzed system is shown in Figure 1. It consists of an oscillating body and two unbalanced rotors fixed on it. The oscillating body of mass $m$ can move in $x-, y$-, and $\psi$ directions, and its movement is described by coordinates $x$, $y$, and $\psi$. The oscillating body is connected to the refuge by four linear springs installed symmetrically with stiffness coefficient $k_{x} / 2$ and damping coefficient $f_{x} / 2$ in $x$-direction, with stiffness coefficient $k_{y} / 2$ and damping coefficient $f_{y} / 2$ in $y$-direction, with stiffness coefficient $k_{\psi}$ and damping coefficient $f_{\psi}$ in $\psi$-direction. Each unbalanced rotor preserves an eccentric length $r$ and a mass $m_{i}$, where $i=1,2$, mounted at two identical induction motors' shaft, respectively. The motion of each rotor is described by angle $\varphi_{i}$. Parameter $T_{e i}$ represents the electromagnetic moment, which provides the energy needed to compensate the energy dissipation due to viscous forces and to keep the rotor rotation. The point $o^{\prime \prime}$ is the mass center of the oscillating body. Points $o_{1}$ and $o_{2}$ are the rotating centers of the unbalanced rotors 1 and 2 , respectively. Coordinate $o x y$ is the fixed frame; coordinate $o^{\prime} x^{\prime} y^{\prime}$ is the moving frame; coordinate $o^{\prime} x^{\prime \prime} y^{\prime \prime}$ is the rotation frame. The dynamic equation of the system can be given as follows [5]:

$$
\begin{aligned}
& J_{0 i} \ddot{\varphi}_{i}+f_{i} \dot{\varphi}_{i}+m_{i} r \ddot{y} \cos \varphi_{i}+(-1)^{i} m_{i} r \ddot{x} \sin \varphi_{i}+(-1)^{i} \\
& \cdot m_{i} l_{i} \ddot{\psi} \cos \left(\varphi_{i}+\beta_{i}\right)+(-1)^{i} m_{i} r l_{i} \dot{\psi}^{2} \sin \left(\varphi_{i}+\beta_{i}\right) \\
& =T_{e i}, \quad(i=1,2), \\
& \left(m+\sum_{i=1}^{2} m_{i}\right) \ddot{x}+f_{x} \dot{x}+k_{x} x \\
& =\sum_{i=1}^{2}(-1)^{i+1} m_{i} r\left(\dot{\varphi}_{i}^{2} \cos \varphi_{i}+\ddot{\varphi}_{i} \sin \varphi_{i}\right),
\end{aligned}
$$




$$
\begin{aligned}
& \left(m+\sum_{i=1}^{2} m_{i}\right) \ddot{y}+f_{y} \dot{y}+k_{y} y \\
& =\sum_{i=1}^{2} m_{i} r\left(\dot{\varphi}_{i}^{2} \sin \varphi_{i}-\ddot{\varphi}_{i} \cos \varphi_{i}\right), \\
& J \ddot{\psi}+f_{\psi} \dot{\psi}+k_{\psi} \psi=\sum_{i=1}^{2}(-1)^{i} \\
& \cdot m_{i} r l_{i}\left[\dot{\varphi}_{i}^{2} \sin \left(\varphi_{i}-\beta_{i}\right)-\ddot{\varphi}_{i} \cos \left(\varphi_{i}-\beta_{i}\right)\right] .
\end{aligned}
$$

In (1) (4), $l_{i}$ is the distance between the rotating center of the unbalanced rotors and the mass center of the oscillating body; $\beta_{i}$ is the angle between $o^{\prime \prime} o_{i}$ and $x$-direction $\left(\beta_{1}=\pi-\beta_{2}\right) ; J$ is the moment of inertia of the whole vibrating system about the mass center of the oscillating body; $J_{0 i}$ is the moment of inertia of the unbalanced rotor $i ;(\bullet)$ and $(\bullet)$ represent $d(\bullet) / d t$ and $d^{2}(\bullet) / d t^{2}$, respectively.

\section{Energy Balance of the System}

In synchronous state, multiplying (1) by the angular velocity of the rotor and integrating it over the period $T$, we obtain the equation of the energy balance:

$$
\begin{aligned}
& \int_{0}^{T} J_{0 i} \ddot{\varphi}_{i} \dot{\varphi}_{i} d t+\int_{0}^{T} f_{i} \dot{\varphi}_{i} \dot{\varphi}_{i} d t+\int_{0}^{T} m_{i} r \ddot{y} \dot{\varphi}_{i} \cos \varphi_{i} d t \\
& \quad+\int_{0}^{T}(-1)^{i} m_{i} r \ddot{x} \dot{\varphi}_{i} \sin \varphi_{i} d t \\
& \quad+\int_{0}^{T}(-1)^{i} m_{i} l_{i} \ddot{\psi} \dot{\varphi}_{i} \cos \left(\varphi_{i}+\beta_{i}\right) d t \\
& \quad+\int_{0}^{T}(-1)^{i} m_{i} r l_{i} \dot{\psi}^{2} \dot{\varphi}_{i} \sin \left(\varphi_{i}+\beta_{i}\right) d t \\
& =\int_{0}^{T} T_{e i} \dot{\varphi}_{i} d t, \quad(i=1,2) .
\end{aligned}
$$

The first component of (5) indicates the energy produced by the inertia force where the induction motors act on the $i$ th rotor. However, the value of the energy in synchronous state is approximately zero:

$$
W_{i}^{\mathrm{INERT}}=\int_{0}^{T} J_{0 i} \ddot{\varphi}_{i} \dot{\varphi}_{i} d t \approx 0 .
$$

The second component of (5) represents energy dissipated by the $i$ th rotor in the joints between the roller and the shaft of the induction motor:

$$
W_{i}^{\mathrm{DAMP}}=\int_{0}^{T} f_{i} \dot{\varphi}_{i} \dot{\varphi}_{i} d t
$$

The next four components describe the energy transferred by the $i$ th rotor to the oscillating body:

$$
\begin{aligned}
W_{i}^{\mathrm{SYS}}= & \int_{0}^{T} m_{i} r \ddot{y} \dot{\varphi}_{i} \cos \varphi_{i} d t+\int_{0}^{T}(-1)^{i} m_{i} r \ddot{x} \dot{\varphi}_{i} \sin \varphi_{i} d t \\
& +\int_{0}^{T}(-1)^{i} m_{i} l_{i} \ddot{\psi} \dot{\varphi}_{i} \cos \left(\varphi_{i}+\beta_{i}\right) d t \\
& +\int_{0}^{T}(-1)^{i} m_{i} r l_{i} \dot{\psi}^{2} \dot{\varphi}_{i} \sin \left(\varphi_{i}+\beta_{i}\right) d t
\end{aligned}
$$

Integral on the right hand side of (5) describes the part of the work performed by the induction motors, that is, the part of this work which is connected with the motion of rotors:

$$
W_{i}^{\mathrm{DRIVE}}=\int_{0}^{T} T_{e i} \dot{\varphi}_{i} d t .
$$

Substituting (6) (9) into (5) one gets the energy balance of the $i$ th rotor:

$$
W_{i}^{\mathrm{SYS}}+W_{i}^{\mathrm{DAMP}}=W_{i}^{\mathrm{DRIVE}} \quad(i=1,2) .
$$

Adding the energy balance of the two rotors according to (10), we have

$$
\sum_{i=1}^{2} W_{i}^{\mathrm{SYS}}+\sum_{i=1}^{2} W_{i}^{\mathrm{DAMP}}=\sum_{i=1}^{2} W_{i}^{\mathrm{DRIVE}}
$$

The total synchronization energy, that is, the energy transferred by the rotors to the oscillating body, is given by

$$
\begin{aligned}
& \sum_{i=1}^{2} W_{i}^{\mathrm{SYS}}=\sum_{i=1}^{2}\left[\int_{0}^{T} m_{i} r \ddot{y} \dot{\varphi}_{i} \cos \varphi_{i} d t\right. \\
& \quad+\int_{0}^{T}(-1)^{i} m_{i} r \ddot{x} \dot{\varphi}_{i} \sin \varphi_{i} d t \\
& \quad+\int_{0}^{T}(-1)^{i} m_{i} l_{i} \ddot{\psi} \dot{\varphi}_{i} \cos \left(\varphi_{i}+\beta_{i}\right) d t \\
& \left.\quad+\int_{0}^{T}(-1)^{i} m_{i} r l_{i} \dot{\psi}^{2} \dot{\varphi}_{i} \sin \left(\varphi_{i}+\beta_{i}\right) d t\right] .
\end{aligned}
$$

The total energy dissipated in the joint connecting rotors is given by

$$
\sum_{i=1}^{2} W_{i}^{\mathrm{DAMP}}=\sum_{1}^{2} \int_{0}^{T} f_{i} \dot{\varphi}_{i} \dot{\varphi}_{i} d t
$$

Moreover, the total energy of the two induction motors is given by

$$
\sum_{i=1}^{2} W_{i}^{\mathrm{DRIVE}}=\sum_{i=1}^{2} \int_{0}^{T} T_{e i} \dot{\varphi}_{i} d t
$$

Next multiplying (2) by the velocity of the oscillating body in the $x$-direction and integrating it over the period $T$, we 
obtain the equation of the energy balance of the oscillating body:

$$
\begin{gathered}
\int_{0}^{T}\left(m+\sum_{i=1}^{2} m_{i}\right) \ddot{x} \dot{x} d t+\int_{0}^{T} f_{x} \dot{x} \dot{x} d t+\int_{0}^{T} k_{x} x \dot{x} d t \\
=\sum_{i=1}^{2} \int_{0}^{T}(-1)^{i+1} m_{i} r\left(\dot{\varphi}_{i}^{2} \cos \varphi_{i}+\ddot{\varphi}_{i} \sin \varphi_{i}\right) \dot{x} d t .
\end{gathered}
$$

The first component on the left hand side of (15) represents the increase of the kinematic energy of the oscillating body and two rotors during the period $T$, which should be equal to zero (because of the periodic motion of oscillating body in the synchronous state):

$$
W_{x}^{\mathrm{INERT}}=\int_{0}^{T}\left(m+\sum_{i=1}^{2} m_{i}\right) \ddot{x} \dot{x} d t=0 .
$$

The next component represents the dissipated energy in the spring damp $f_{x}$ in the $x$-direction:

$$
W_{x}^{\mathrm{DAMP}}=\int_{0}^{T} f_{x} \dot{x} \dot{x} d t
$$

The last component on the left hand side standing for the work performed by the force in the springs, due to the potential character of the force, is equal to zero:

$$
W_{x}^{\mathrm{POT}}=\int_{0}^{T} k_{x} x \dot{x} d t=0 .
$$

On the right side of (15) it gives the resultant force of the two rotors acting on the oscillating body in $x$-direction:

$$
W_{x}^{\mathrm{SYS}}=\sum_{i=1}^{2} \int_{0}^{T}(-1)^{i+1} m_{i} r\left(\dot{\varphi}_{i}^{2} \cos \varphi_{i}+\ddot{\varphi}_{i} \sin \varphi_{i}\right) \dot{x} d t .
$$

Substituting (16) (19) into (15), the energy balance of the oscillating body in $x$-direction can be written as follows:

$$
W_{x}^{\mathrm{DAMP}}=W_{x}^{\mathrm{SYS}} \text {. }
$$

Then multiplying (3) by the velocity of the oscillating body in the $y$-direction and integrating it over the period $T$, we obtain the equation of the energy balance of the oscillating body:

$$
\begin{gathered}
\int_{0}^{T}\left(m+\sum_{i=1}^{2} m_{i}\right) \ddot{y} \dot{y} d t+\int_{0}^{T} f_{y} \dot{y} \dot{y} d t+\int_{0}^{T} k_{y} y \dot{y} d t \\
=\int_{0}^{T} \sum_{i=1}^{2} m_{i} r_{i} \dot{y}\left(\dot{\varphi}_{i}^{2} \sin \varphi_{i}-\ddot{\varphi}_{i} \cos \varphi_{i}\right) d t .
\end{gathered}
$$

The first component on the left hand side of (21) represents the increase of the kinematic energy of the oscillating body and both rotors during the period $T$, which should be equal to zero (because of the periodic motion of the oscillating body in the synchronous state):

$$
W_{y}^{\mathrm{INERT}}=\int_{0}^{T}\left(m+\sum_{i=1}^{2} m_{i}\right) \ddot{y} \dot{y} d t=0 .
$$

The next component represents the dissipated energy in the spring damp $f_{y}$ in the $y$-direction:

$$
W_{y}^{\text {DAMP }}=\int_{0}^{T} f_{y} \dot{y} \dot{y} d t .
$$

The last component on the left hand side standing for the work performed by the force in the springs in the $y$-direction, due to the potential character of the force, is equal to zero:

$$
W_{y}^{\mathrm{POT}}=\int_{0}^{T} k_{y} y \dot{y} d t=0 .
$$

On the right side of (21) it gives the resultant force of the two rotors acting on the oscillating body in $y$-direction:

$$
W_{y}^{\mathrm{SYS}}=\int_{0}^{T} \sum_{i=1}^{2} m_{i} r_{i} \dot{y}\left(\dot{\varphi}_{i}^{2} \sin \varphi_{i}-\ddot{\varphi}_{i} \cos \varphi_{i}\right) d t
$$

Substituting (22) (25) into (21) the energy balance of the oscillating body in $y$-direction can be rewritten as follows:

$$
W_{y}^{\text {SYS }}=W_{y}^{\text {DAMP }} .
$$

At last, multiplying (3) by the velocity of the oscillating body in the $\psi$-direction and integrating it over the period $T$, we obtain the equation of the energy balance of the oscillating body:

$$
\begin{aligned}
& \int_{0}^{T} J \ddot{\psi} \dot{\psi} d t+\int_{0}^{T} f_{\psi} \dot{\psi} \dot{\psi} d t+\int_{0}^{T} k_{\psi} \psi \dot{\psi} d t \\
& \quad=\sum_{i=1}^{2} \int_{0}^{T}(-1)^{i} \\
& \quad \cdot m_{i} r l_{i} \dot{\psi}\left[\dot{\varphi}_{i}^{2} \sin \left(\varphi_{i}-\beta_{i}\right)-\ddot{\varphi}_{i} \cos \left(\varphi_{i}-\beta_{i}\right)\right] d t .
\end{aligned}
$$

The first component on the left hand side of (27) represents the increase of the kinematic energy of the oscillating body and rotors during the period $T$, which should be equal to zero (because of the periodic motion of oscillating body):

$$
W_{\psi}^{\mathrm{INERT}}=\int_{0}^{T} J \ddot{\psi} \dot{\psi} d t=0 .
$$

The next component represents the dissipated energy in the spring damp $f_{\psi}$ in the $\psi$-direction:

$$
W_{\psi}^{\text {DAMP }}=\int_{0}^{T} f_{\psi} \dot{\psi} \dot{\psi} d t .
$$

The last component on the left hand side standing for the work performed by the force moment in the spring in the $\psi$ direction, due to the potential character of the force, is equal to zero:

$$
W_{\psi}^{\mathrm{POT}}=\int_{0}^{T} k_{\psi} \psi \dot{\psi} d t=0 .
$$


On the right side of (27) it gives the resultant moment of the two rotors acting on the oscillating body in $\psi$-direction:

$$
\begin{aligned}
& W_{\psi}^{\mathrm{SYS}}=\sum_{i=1}^{2} \int_{0}^{T}(-1)^{i} \\
& \cdot m_{i} r l_{i} \dot{\psi}\left[\dot{\varphi}_{i}^{2} \sin \left(\varphi_{i}-\beta_{i}\right)-\ddot{\varphi}_{i} \cos \left(\varphi_{i}-\beta_{i}\right)\right] d t .
\end{aligned}
$$

Substituting (28) (31) into (27) the energy balance of the oscillating body in $\psi$-direction can be written as follows:

$$
W_{\psi}^{\text {DAMP }}=W_{\psi}^{\text {SYS }} \text {. }
$$

Adding together (20), (26), and (32) we obtain the energy balance of the oscillating body in $x_{-}^{-}, y^{-}$, and $\psi$-directions:

$$
\sum_{j=x, y, \psi} W_{j}^{\mathrm{DAMP}}=\sum_{j=x, y, \psi} W_{j}^{\mathrm{SYS}} .
$$

\section{Synchronization Condition and Synchronous Stability Criterion of the System}

4.1. Synchronization Condition. Adding (11) and (33) we get the energy balance of the whole system in the following form:

$$
\begin{aligned}
& \sum_{i=1}^{2} W_{i}^{\mathrm{INERT}}+\sum_{i=1}^{2} W_{i}^{\mathrm{SYS}}+\sum_{i=1}^{2} W_{i}^{\mathrm{DAMP}}+\sum_{j=x, y, \psi} W_{j}^{\mathrm{DAMP}} \\
& =\sum_{i=1}^{2} W_{i}^{\mathrm{DRIVE}}+\sum_{j=x, y, \psi} W_{j}^{\mathrm{SYS}} \cdot
\end{aligned}
$$

During the synchronous state oscillations the energy supplied by the motors is dissipated by the dampers: that is,

$$
\sum_{i=1}^{2} W_{i}^{\mathrm{DAMP}}+\sum_{j=x, y, \psi} W_{j}^{\mathrm{DAMP}}=\sum_{i=1}^{2} W_{i}^{\mathrm{DRIVE}}
$$

with

$$
\sum_{i=1}^{2} W_{i}^{\text {SYS }}=\sum_{j=x, y, \psi} W_{j}^{\text {SYS }}
$$

Substituting (33) into (35) and considering (36), the energy balance of the whole system during the steady periodic oscillations (or during the synchronous state) can be written by

$$
\sum_{i=1}^{2} W_{i}^{\mathrm{SYS}}+\sum_{i=1}^{2} W_{i}^{\mathrm{DAMP}}=\sum_{i=1}^{2} W_{i}^{\mathrm{DRIVE}}
$$

Equation (37) describes the energy balance of the whole system within the energy transmission. It can be said that the sum of the synchronization energy and the energy dissipated by the rotors in the joints is equal to the energy produced by the induction motors.
In actual engineering applications, the same type of motors keeps the different electrical characteristics as manufacturing tolerances. According to (10) and (37), a subtraction operation on the balance equation of the two rotors, the equation of the energy difference of the system in the synchronous state is given by:

$$
\prod_{i=1}^{2} W_{i}^{\mathrm{SYS}}+\prod_{i=1}^{2} W_{i}^{\mathrm{DAMP}}=\prod_{i=1}^{2} W_{i}^{\mathrm{DRIVE}} .
$$

We assume that the average phase angular and rotation velocity of the rotors are $\varphi$ and $\omega_{m}$, respectively, when the vibrating system operates in synchronous state. We obtain

$$
\begin{aligned}
& \dot{\varphi}=\omega_{m}=\text { constant, } \\
& \ddot{\varphi}=0 .
\end{aligned}
$$

As shown in Figure 1, assuming the average phase and the phase difference of the two unbalanced rotors to be $\varphi$ and $2 \alpha$, respectively, then we have

$$
\begin{aligned}
& \varphi_{1}=\varphi+\alpha, \\
& \varphi_{2}=\varphi-\alpha .
\end{aligned}
$$

According to (2) (4) and (39), in the synchronous state the response of the vibrating system can be expressed as follows:

$$
\begin{aligned}
x & =-r r_{m} \mu_{x}\left[\cos \left(\varphi+\alpha+\gamma_{x}\right)-\eta \cos \left(\varphi-\alpha+\gamma_{x}\right)\right] \\
y & =-r r_{m} \mu_{y}\left[\sin \left(\varphi+\alpha+\gamma_{y}\right)+\eta \sin \left(\varphi-\alpha+\gamma_{y}\right)\right] \\
\psi & =-r r_{m} \mu_{\psi}\left[-\frac{r_{l 1} \sin \left(\varphi+\alpha+\beta_{i}+\gamma_{\psi}\right)}{l_{e}}\right. \\
& \left.+\frac{\eta r_{l} r_{l 1} \sin \left(\varphi-\alpha+\beta_{i}+\gamma_{\psi}\right)}{l_{e}}\right],
\end{aligned}
$$

where $\omega_{n x}=\sqrt{k_{x} / M}, \omega_{n y}=\sqrt{k_{y} / M}, \omega_{n \psi}=\sqrt{k_{\psi} / J}, \xi_{n x}=$ $f_{x} / \sqrt{4 k_{x} M}, \xi_{n x}=f_{x} / \sqrt{4 k_{x} M}, \xi_{n \psi}=f_{\psi} / \sqrt{4 k_{\psi} J}, \mu_{x}=1 /(1-$ $\left.\omega_{n x}^{2} / \omega_{m}^{2}\right), \mu_{y}=1 /\left(1-\omega_{n y}^{2} / \omega_{m}^{2}\right), \mu_{\psi}=1 /\left(1-\omega_{n \psi}^{2} / \omega_{m}^{2}\right), r_{m}=$ $m_{1} / M, \eta=m_{1} / m_{2}, l_{e}=\sqrt{J / M}, r_{l 1}=l_{1} / l_{e}, r_{l 2}=l_{2} / l_{e}, \gamma_{j}=$ $\left[2 \xi_{n j}\left(\omega_{n j} / \omega_{m}\right)\right] /\left[1-\left(\omega_{n j} / \omega_{m}\right)^{2}\right],(j=x, y, \psi)$, and $r_{l}=l_{2} / l_{1}$. $\xi_{n j}$ is the damping ratio of the springs in the $j$-direction $\left(\xi_{n j} \leq\right.$ 0.07 in this paper). Differentiating the formulas in (41) with respect to time $t$, we can obtain $\ddot{x}, \ddot{y}, \dot{\psi}$, and $\ddot{\psi}$.

In the synchronous state of the two rotors, the phase angle difference $\varphi_{1}-\varphi_{2}=2 \alpha$ should be a constant and independent of the initial conditions. Therefore, the dynamic parameters of the vibrating system should satisfy the energy balance equation and the energy difference equation, that is, 
(37) and (38). Substituting those dynamic parameters above into (37) and (38), we have

$$
\begin{aligned}
& \sum_{i=1}^{2} \int_{0}^{T} m_{i} r \omega_{m} \ddot{y} \cos \varphi_{i} d t+\int_{0}^{T}(-1)^{i} m_{i} r \omega_{m} \ddot{x} \sin \varphi_{i} d t \\
& \quad+\int_{0}^{T}(-1)^{i} m_{i} r \omega_{m} \ddot{\psi} \cos \left(\varphi_{i}+\beta_{i}\right) d t \\
& \quad+\int_{0}^{T}(-1)^{i} m_{i} r \omega_{m} l_{i} \dot{\psi}^{2} \sin \left(\varphi_{i}+\beta_{i}\right) d t \\
& \quad+\int_{0}^{T} f_{i} \omega_{m}^{2} d t=\sum_{i=1}^{2} \int_{0}^{T} T_{e i} \omega_{m} d t, \\
& \quad+\int_{i=1}^{T} m_{i} r \omega_{m} \ddot{y} \cos \varphi_{i} d t+\int_{0}^{T}(-1)^{i} m_{i} r \omega_{m} \ddot{x} \sin \varphi_{i} d t \\
& \quad+\int_{0}^{T}(-1)^{i} m_{i} r \omega_{m} l_{i} \ddot{\psi} \cos \left(\varphi_{i}+\beta_{i}\right) d t \\
& \quad+\int_{0}^{T}(-1)^{i} m_{i} r \omega_{m} l_{i} \dot{\psi}^{2} \sin \left(\varphi_{i}+\beta_{i}\right) d t \\
& \quad f_{i} \omega_{m}^{2} d t=\prod_{i=1}^{2} \int_{0}^{T} T_{e i} \omega_{m} d t .
\end{aligned}
$$

Considering $\omega_{m 0} d t=d \varphi$, (42) can be rewritten as

$$
\begin{aligned}
& \sum_{i=1}^{2} \int_{0}^{2 \pi} m_{i} r \ddot{y} \cos \varphi_{i} d \varphi+\int_{0}^{2 \pi}(-1)^{i} m_{i} r \ddot{x} \sin \varphi_{i} d \varphi \\
& \quad+\int_{0}^{2 \pi}(-1)^{i} m_{i} r \ddot{\psi} \cos \left(\varphi_{i}+\beta_{i}\right) d \varphi \\
& \quad+\int_{0}^{2 \pi}(-1)^{i} m_{i} r l_{i} \dot{\psi}^{2} \sin \left(\varphi_{i}+\beta_{i}\right) d \varphi \\
& \quad+\int_{0}^{2 \pi} f_{i} \omega_{m} d \varphi=\sum_{i=1}^{2} \int_{0}^{2 \pi} T_{e i} d \varphi, \\
& \quad \prod_{i=1}^{2} \int_{0}^{2 \pi} m_{i} r \ddot{y} \cos \varphi_{i} d \varphi+\int_{0}^{2 \pi}(-1)^{i} m_{i} r \ddot{x} \sin \varphi_{i} d \varphi \\
& \quad+\int_{0}^{2 \pi}(-1)^{i} m_{i} r \ddot{\psi} \cos \left(\varphi_{i}+\beta_{i}\right) d \varphi \\
& \quad+\int_{0}^{2 \pi}(-1)^{i} m_{i} r l_{i} \dot{\psi}^{2} \sin \left(\varphi_{i}+\beta_{i}\right) d \varphi \\
& \quad+\int_{0}^{2 \pi} f_{i} \omega_{m} d \varphi=\prod_{i=1}^{2} \int_{0}^{2 \pi} T_{e i} d \varphi .
\end{aligned}
$$

Substituting $\ddot{x}, \ddot{y}, \dot{\psi}$, and $\ddot{\psi}$ into (43) and integrating them over $\varphi=2 \pi$, we have

$$
\begin{aligned}
& \frac{1}{2} m_{1} r^{2} \omega_{m}^{2}\left[T_{S+}+2 T_{S} \cos \left(2 \alpha+\theta_{S}\right)\right]+\left(f_{1}+f_{2}\right) \omega_{m} \\
& \quad=T_{e 1}+T_{e 2} \\
& \frac{1}{2} m_{1} r^{2} \omega_{m}^{2}\left[T_{S-}+2 T_{C} \sin \left(2 \alpha+\theta_{C}\right)\right]+\left(f_{1}-f_{2}\right) \omega_{m} \\
& \quad=T_{e 1}-T_{e 2},
\end{aligned}
$$

where

$$
\begin{aligned}
& T_{S+}=T_{S 1}+T_{S 2}, \\
& T_{S_{-}}=T_{S 1}-T_{S 2} ; \\
& T_{S 1}=r_{m}\left(\mu_{y} \sin \gamma_{y}+\mu_{x} \sin \gamma_{x}+r_{l 1}^{2} \mu_{\psi} \sin \gamma_{\psi}\right), \\
& T_{S 2}=\eta^{2} r_{m}\left(\mu_{y} \sin \gamma_{y}+\mu_{x} \sin \gamma_{x}+r_{l}^{2} r_{l 1}^{2} \mu_{\psi} \sin \gamma_{\psi}\right) ; \\
& T_{S}=\eta r_{m}\left|a_{S}\right|, \\
& T_{C}=\eta r_{m}\left|a_{C}\right| ; \\
& a_{S}=\mu_{y} \sin \gamma_{y}-\mu_{x} \sin \gamma_{x}-\mu_{\psi} r_{l 1} r_{l 2} \sin \gamma_{\psi}, \\
& a_{C}=-\mu_{y} \cos \gamma_{y}+\mu_{x} \cos \gamma_{x}+\mu_{\psi} r_{l} r_{l 1}^{2} \cos \gamma_{\psi} ; \\
& \theta_{S}= \begin{cases}0, & a_{S} \geq 0 \\
\pi, & a_{S}<0,\end{cases} \\
& \theta_{C}= \begin{cases}0, & a_{C} \geq 0 \\
\pi, & a_{C}<0 .\end{cases}
\end{aligned}
$$

The first formula of (44) is the equation of torque balance of the vibrating system in the synchronous state, which serves to find the approximation of angular velocity $\omega_{m}$. Moreover, the second formula of (44) is balance equation of the torque difference of the two rotors in the synchronous state, which serves to determine the approximation of stable phase difference $2 \alpha$.

Specifying $T_{V}$ as the vibratory torque of the vibrating system, we have

$$
T_{V}=m_{1} r^{2} \omega_{m}^{2} T_{C}
$$

Then assigning $T_{E}$ as the excessive torque of the rotors, we have

$$
T_{E}=T_{\text {Residual1 }}-T_{\text {Residual2 }}
$$

where $T_{\text {Residual1 }}$ and $T_{\text {Residual2 }}$ represent the residual torques of rotor 1 and 2, respectively. They can be written as

$$
\begin{aligned}
& T_{\text {Residual1 }}=T_{e 1}-f_{1} \omega_{m}-\frac{1}{2} m_{1} r^{2} \omega_{m}^{2} T_{S 1}, \\
& T_{\text {Residual2 }}=T_{e 2}-f_{2} \omega_{m}-\frac{1}{2} m_{1} r^{2} \omega_{m}^{2} T_{S 2} .
\end{aligned}
$$


Rewriting the second formula of (44), we can obtain

$$
2 \alpha=\arcsin \frac{T_{E}}{T_{V}}-\theta_{C} .
$$

To ensure the existence of the solution to $2 \alpha$, we should have $\left|\sin 2 \alpha+\theta_{C}\right| \leq 1$. Therefore, the vibratory torque of the vibrating system $T_{V}$ must be equal to or greater than the absolute value of the excessive torque of the rotors $\left|T_{E}\right|$. This is the synchronization condition of the vibrating system, and it could be expressed as

$$
T_{V} \geq\left|T_{E}\right|
$$

In this case, the synchronous angular velocity $\omega_{m}$ and the phase difference $2 \alpha$ of the two rotors can be solved to (49) with a numeric method. If the excessive torque of the motors approaches to zero (i.e., $\left|T_{E}\right| \rightarrow 0$ ), the residual electromagnetic torque of motor 1 is approximately equal to that of motor 2. In this case, the two rotors can easily operate synchronously.

4.2. Synchronization Stability Criterion. The stability criterion of the synchronous state of the two rotors in the vibrating system will be discussed in the following section by solving a disturbance differential equation. Introducing disturbance parameters $\Delta \ddot{\alpha}_{1}, \Delta \dot{\alpha}_{1}, \Delta \alpha_{1}, \Delta \ddot{\alpha}_{2}, \Delta \dot{\alpha}_{2}$, and $\Delta \alpha_{2}$ into (1) and substituting them into (43), the disturbance differential equation of the synchronous state of the two rotors can be obtained by

$$
\begin{aligned}
& J_{01} \Delta \ddot{\alpha}_{1}+f_{1} \Delta \dot{\alpha}_{1}+\frac{1}{2} \eta m_{1} r^{2} \omega_{m}^{2} r_{m}\left\{\left[-\mu_{y} \sin \left(2 \alpha-r_{y}\right)\right.\right. \\
& \left.+\mu_{x} \sin \left(2 \alpha-r_{x}\right)+\mu_{\psi} r_{l 2}^{2} \sin \left(2 \alpha-r_{\psi}\right)\right] \cos \left(\Delta \alpha_{1}\right. \\
& \left.\quad-\Delta \alpha_{2}\right)+\left[-\mu_{y} \cos \left(2 \alpha-r_{y}\right)+\mu_{x} \cos \left(2 \alpha-r_{x}\right)\right. \\
& \left.+\mu_{\psi} r_{l 1} r_{l 2} \cos \left(2 \alpha-r_{\psi}\right)\right] \sin \left(\Delta \alpha_{1}-\Delta \alpha_{2}\right)+\mu_{y} \\
& +\sin \left(2 \alpha-r_{y}\right)-\mu_{x} \sin \left(2 \alpha-r_{x}\right)-\mu_{\psi} r_{l 2}^{2} \sin (2 \alpha \\
& \left.\left.\quad-r_{\psi}\right)\right\}=0, \\
& J_{02} \Delta \ddot{\alpha}_{2}+f_{2} \Delta \dot{\alpha}_{2}+\frac{1}{2} \eta m_{1} r^{2} \omega_{m}^{2} r_{m}\left\{\left[\mu_{y} \sin \left(2 \alpha+r_{y}\right)\right.\right. \\
& \left.+\mu_{x} \sin \left(2 \alpha+r_{x}\right)-\mu_{\psi} r_{l 2}^{2} \sin \left(2 \alpha+r_{\psi}\right)\right] \cos \left(\Delta \alpha_{1}\right. \\
& \left.-\Delta \alpha_{2}\right)+\left[\mu_{y} \cos \left(2 \alpha+r_{y}\right)-\mu_{x} \cos \left(2 \alpha+r_{x}\right)\right. \\
& \left.-\mu_{\psi} r_{l 1} r_{l 2} \cos \left(2 \alpha+r_{\psi}\right)\right] \sin \left(\Delta \alpha_{1}-\Delta \alpha_{2}\right) \\
& +\mu_{y} \sin \left(2 \alpha+r_{y}\right)-\mu_{x} \sin \left(2 \alpha+r_{x}\right) \\
& \left.+\mu_{\psi} r_{l 2}^{2} \sin \left(2 \alpha+r_{\psi}\right)\right\}=0 .
\end{aligned}
$$

From (51), it is noted that $\left[\Delta \alpha_{1}, \Delta \alpha_{2}\right]^{T}=[0,0]^{T}$ is an equilibrium solution when the two rotors rotate synchronously. Then linearizing (51) around $\left[\Delta \alpha_{1}, \Delta \alpha_{2}\right]^{T}=[0,0]^{T}$ we have

$$
\begin{aligned}
& J_{01} \Delta \ddot{\alpha}_{1}+f_{1} \Delta \dot{\alpha}_{1}+\frac{1}{2} \eta m_{1} r^{2} \omega_{m}^{2} r_{m}\left[-\mu_{y} \cos \left(2 \alpha-r_{y}\right)\right. \\
& \left.+\mu_{x} \cos \left(2 \alpha-r_{x}\right)+\mu_{\psi} r_{l 1} r_{l 2} \cos \left(2 \alpha-r_{\psi}\right)\right]\left(\Delta \alpha_{1}\right. \\
& \left.-\Delta \alpha_{2}\right)=0, \\
& J_{02} \Delta \ddot{\alpha}_{2}+f_{2} \Delta \dot{\alpha}_{12}+\frac{1}{2} \eta m_{1} r^{2} \omega_{m}^{2} r_{m}\left[\mu_{y} \cos \left(2 \alpha+r_{y}\right)\right. \\
& \left.-\mu_{x} \cos \left(2 \alpha+r_{x}\right)-\mu_{\psi} r_{l 1} r_{l 2} \cos \left(2 \alpha+r_{\psi}\right)\right]\left(\Delta \alpha_{1}\right. \\
& \left.-\Delta \alpha_{2}\right)=0 .
\end{aligned}
$$

According to (44), (52) can also be rewritten as

$$
\begin{aligned}
& \Delta \ddot{\alpha}_{1}+\frac{f_{1}}{m_{1} r^{2}} \Delta \dot{\alpha}_{1}+\frac{\eta}{2} \\
& \cdot \omega_{m}^{2}\left[T_{C} \cos \left(2 \alpha+\theta_{C}\right)+T_{S} \sin \left(2 \alpha+\theta_{S}\right)\right] \\
& \cdot\left(\Delta \alpha_{1}-\Delta \alpha_{2}\right)=0 \\
& \Delta \ddot{\alpha}_{2}+\frac{f_{2}}{\eta m_{1} r^{2}} \Delta \dot{\alpha}_{2}+\frac{\eta}{2} \\
& \cdot \omega_{m}^{2}\left[-T_{C} \cos \left(2 \alpha+\theta_{C}\right)+T_{S} \sin \left(2 \alpha+\theta_{S}\right)\right] \\
& \cdot\left(\Delta \alpha_{1}-\Delta \alpha_{2}\right)=0 .
\end{aligned}
$$

When the following parameters of the damping coefficient of the two motor shafts in the system satisfy the condition, that is, $f_{1} \approx f_{2} / \eta$, the disturbance differential equation can be obtained with subtracting each equation of (53):

$$
\Delta \ddot{\alpha}+\frac{f_{1}}{m_{1} r^{2}} \Delta \dot{\alpha}+\eta \omega_{m}^{2}\left[T_{C} \cos \left(2 \alpha+\theta_{C}\right)\right] \Delta \alpha=0,
$$

where $\Delta \ddot{\alpha}=\Delta \ddot{\alpha}_{1}-\Delta \ddot{\alpha}_{2}, \Delta \dot{\alpha}=\Delta \dot{\alpha}_{1}-\Delta \dot{\alpha}_{2}$, and $\Delta \alpha=\Delta \alpha_{1}-\Delta \alpha_{2}$. Symbols $\Delta \ddot{\alpha}, \Delta \dot{\alpha}$, and $\Delta \alpha$ denote acceleration, velocity, and displacement of the phase difference of the two rotors when a disturbance acts on the vibrating system, respectively.

According to the stability theory, the coefficient of the third item of (54) must be positive because the coefficient of the first two items is obviously greater than zero. In this case, the synchronous state of the vibrating system can be stable. As $\eta>0$ and $\omega_{m}^{2}>0$, the criterion of the synchronization stability of the system can be expressed as

$$
T_{C} \cos \left(2 \alpha+\theta_{C}\right)>0 .
$$

In the far-resonant vibrating system we have $T_{C}>0$ by $\mu_{y} \approx \mu_{x}, \cos \gamma_{y} \approx \cos \gamma_{x}, \mu_{\psi}>0, r_{l}>0$ and $\cos \gamma_{\psi}>0$; thus, it leads to $\theta_{C}=0$, and so $\cos \left(2 \alpha+\theta_{C}\right)=\cos 2 \alpha$. In the light of the criterion of the synchronization stability of the system (i.e., (55)), only if parameter $\cos (2 \alpha)>0$ can be satisfied, the stability of the synchronous rotation of the two rotors can be implemented. However, parameter $\cos (2 \alpha)>0$ also indicated that $2 \alpha \in(\pi / 2,-\pi / 2)$ is the interval of the stable phase difference of the two rotors during the synchronous operation. 


\section{Numerical Verification}

5.1. Numerical Discussion for Stable Phase Difference. In our numerical verification, we consider the following values of the system parameters. We assume the parameters of the two motors are identical (i.e., rated power $0.7 \mathrm{Kw}$, rated voltage $220 \mathrm{~V}$, rated frequency $50 \mathrm{~Hz}$, pole pairs 2, stator resistance $0.56 \Omega$, rotor resistance $0.54 \Omega$, stator inductance $0.1 \mathrm{H}$, rotor inductance $0.12 \mathrm{H}$, mutual inductance $0.13 \mathrm{H}$, and the damping coefficient of shafting $0.04 \mathrm{Nm} /(\mathrm{rad} / \mathrm{s}))$. And the parameters of the vibrating system are $M=120 \mathrm{~kg}$, $m_{1}=4 \mathrm{~kg}, f_{x}=0.38 \mathrm{kN} \cdot \mathrm{s} / \mathrm{m}, f_{y}=0.38 \mathrm{kN} \cdot \mathrm{s} / \mathrm{m}, f_{\psi}=$ $0.034 \mathrm{kN} \cdot \mathrm{m} /(\mathrm{rad} / \mathrm{s}), f_{1}=0.003 \mathrm{kN} \cdot \mathrm{m} /(\mathrm{rad} / \mathrm{s}), r=0.05 \mathrm{~m}$, $l_{e}=0.3 \mathrm{~m}, r_{l 1}=1, r_{l}=0 \sim 5, \beta_{1}=45^{\circ}, \beta_{2}=135^{\circ}$, $\eta=0.4 \sim 1, k_{x}=63 \mathrm{kN} / \mathrm{m}, k_{y}=63 \mathrm{kN} / \mathrm{m}, k_{\psi}=5 \mathrm{kN} \cdot \mathrm{m} / \mathrm{rad}$, and $f_{2}=0.003 \mathrm{kN} \cdot \mathrm{m} /(\mathrm{rad} / \mathrm{s})$.

Section 4.1 has given some theoretical analyses in the simplified form on synchronization problem. In this section, we will quantitatively discuss the numerical results of the stable phase difference considering many kinds of features of the vibrating system: the first is that the two nonidentical rotors are installed symmetrically; the second is that the two nonidentical rotors are installed asymmetrically; the last is that the power source of the second rotor is cut off. From (44) (49), the main parameters that influence the stable phase difference are $T_{e 1}, T_{e 2}, f_{1}, f_{2}, T_{S 1}, T_{S 2}$, and $T_{C}$. In addition, $T_{S 1}, T_{S 2}$, and $T_{C}$ are functions of the dimensionless parameters $r_{m}, r_{l}, r_{l 1}, \eta, \mu_{x}, \mu_{y}$, and $\mu_{\psi}$. In a far-resonant vibrating system [8], the value of parameters $\mu_{x}, \mu_{y}$, and $\mu_{\psi}$ ranges from 1.01 to 1.07 , and so we focus on the effect of dimensionless parameters $r_{m}, r_{l}, r_{l 1}$, and $\eta$ on the stable phase difference between the two rotors. When the two identical motors are taken to drive two nonidentical rotors, we have

$$
T_{E}=\frac{1}{2} m_{1} r^{2} \omega_{m}^{2}\left(T_{S 2}-T_{S 1}\right)
$$

We assume here that $T_{e 1}-f_{1} \omega_{m}-\left(T_{e 2}-f_{2} \omega_{m}\right)=0$. Therefore, (49) can be simplified in the form

$$
2 \alpha=\arcsin \frac{T_{S 2}-T_{S 1}}{2 T_{C}} .
$$

According to (57) the stable phase difference between the two rotors can be numerically performed. Figure 2(a) shows the value of the stable phase difference between the two rotors installed symmetrically. It can be seen that the change of parameter $r_{m}$ has little influence on the value of the stable phase difference, and the value gradually approaches to zero with increase of the value of parameter $\eta$. Figure 2(b) shows the stable phase difference between the two rotors installed asymmetrically. It is indicated that the value of the stable phase difference also gradually approaches to zero with increasing the value of parameter $\eta$, and the value is inversely proportional to parameter $r_{l}$. To sum up, although parameters $\eta, r_{m}$, and $r_{l}$ are changed suitably in the vibrating system, the phase difference will be located at vicinity of zero degree.
We assume $T_{e 2}=0$ and $f_{2} \omega_{m}-f_{1} \omega_{m} \approx 0$ when the power source of the second rotors is cut off in synchronous state. Therefore, (49) can be simplified in the form

$$
2 \alpha=\arcsin \frac{2 T_{e 1}+m_{1} r^{2} \omega_{m}^{2}\left(T_{S 2}-T_{S 1}\right)}{2 m_{1} r^{2} \omega_{m}^{2} T_{C}} .
$$

According to [8], when an induction motor operates with synchronization velocity $\omega_{m}$, its electromagnetic torque $T_{e i}$ can be simplified as

$$
T_{e i}=n_{p}^{2} \frac{L_{m i}^{2} U_{S 0}^{2}}{L_{s i}^{2} \omega_{s} R_{r i}}\left(\omega_{s}-n_{p} \omega_{m}\right),
$$

where $L_{m i}$ is the mutual inductance of the $i$ th induction motor; $L_{s i}$ is stator inductance of the $i$ th induction motor; $n_{p}$ is the number of pole pairs of the induction motor; $\omega_{s}$ is synchronous electric angular velocity; $R_{r i}$ is the rotor resistance of the $i$ th induction motor; $U_{S 0}$ is the amplitude of the stator voltage vector.

Subsisting the above given motors' parameters into (59), we can compute the value of parameter $T_{e i}$. Figure $2(\mathrm{c})$ shows the stable phase difference between the two rotors in the power-cutting state (suppose that the synchronous velocity $\omega_{m}$ changes in the interval 156-157 rad/s and the electromagnetic torque $T_{e 1}$ ranges in the interval $0.5-0.7 \mathrm{~N} \cdot \mathrm{m}$ ). It can be seen that the stable phase difference in the power-cutting state is larger than the power-supplying state. Moreover, the value of the stable phase difference is inversely proportional to parameters $r_{l}$ and $\eta$.

5.2. Computer Simulations. Further analyses have been performed by numerical simulations. Our results have been obtained by numerical integration (by 4 th order Runge-Kutta method) of (1) (4). The following examples are simulated to confirm the main results of the above theoretical analysis.

5.2.1. Synchronization in Power-Supplying and Power-Cutting States. In calculations for synchronization of the two rotors in power-supplying and power-cutting states, we consider the following values of the dynamics parameters (identical mass and symmetric location of the two rotors): $r_{l 1}=$ $1.0, r_{l}=1.0$, and $\eta=1$, and other parameters are the same as the aforementioned. In the power-cutting state, the implementation of the synchronization of the two rotors should go through two operation steps. Firstly, the two rotors connected with the induction motors are supplied with the same power sources at the same time, which could be called as power-supplying stage; secondly, the power of one of the rotors is cut off during the synchronization operation, and we could call the step as power-cutting stage. An example of the synchronous operation of the two rotors in the power-cutting state is performed with numerical simulations. Here the two rotors are supplied with power source at first $2.5 \mathrm{~s}$, and the power source on rotor 2 is cut off at $2.5 \mathrm{~s}$.

Figures 3(a)-3(c) represent rotational velocities, torques, and phase difference of the two rotors when the two different states are implemented in the system, respectively. In the 


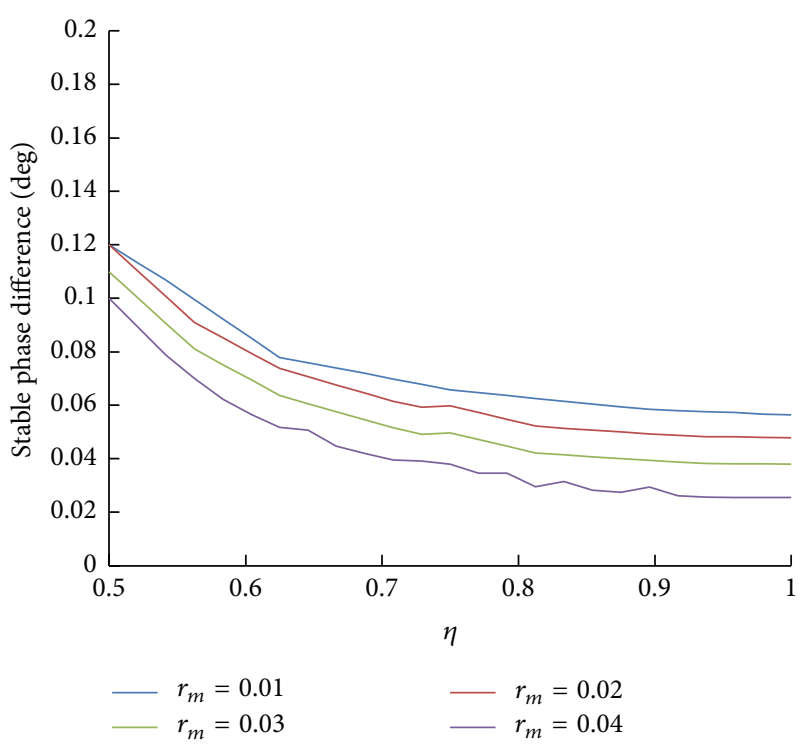

(a)

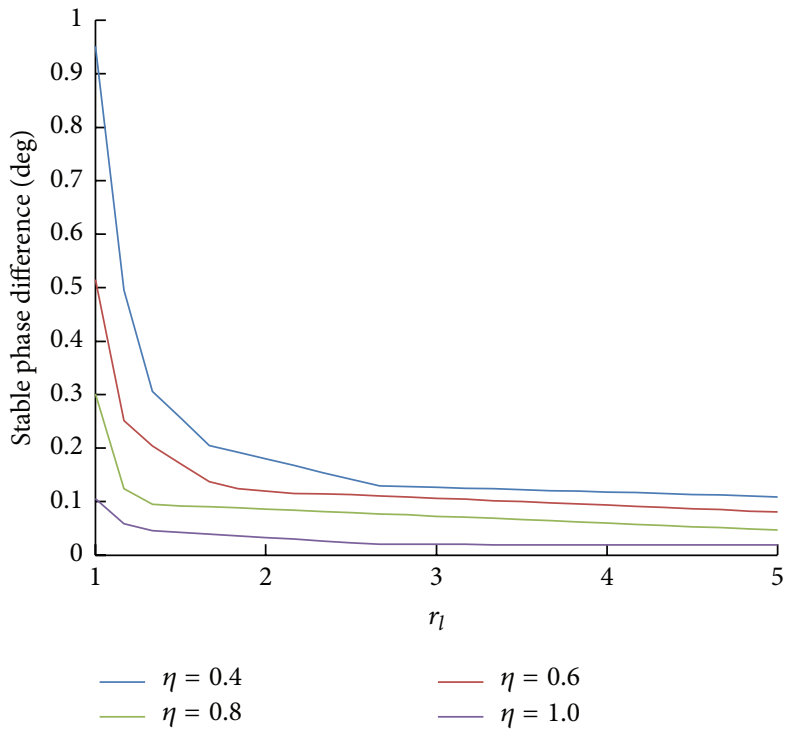

(b)

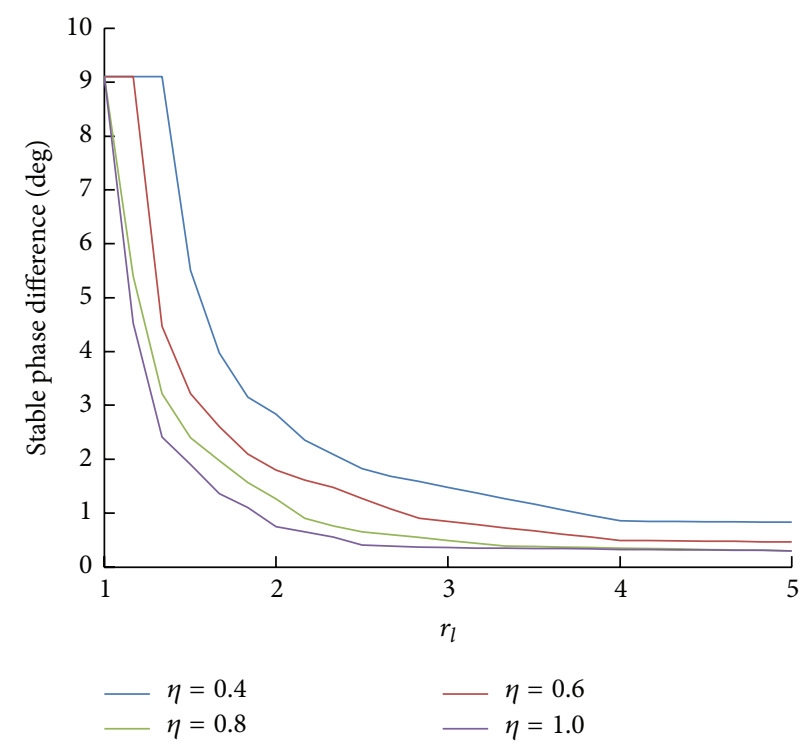

(c)

FIGURE 2: The stable phase difference with different features: (a) $r_{l 1}=1.0$ and $r_{l}=1.0$; (b) $r_{l 1}=1.0$ and $r_{l}=0 \sim 5.0$; (c) $r_{l 1}=1.0, r_{m}=0.15$, $T_{e 1}=1.4$, and $T_{e 2}=0$.

power-supplying stage, the synchronous rotational velocity $\omega_{m}$ of the rotors stabilizes at vicinity of $156.6 \mathrm{rad} / \mathrm{s}$; the electromagnetic torque of the induction motors is approximately equal to $0.7 \mathrm{~N} \cdot \mathrm{m}$; the stable phase difference between the two rotors $2 \alpha$ is $0.5^{\circ}$. In the power-cutting stage, the synchronous rotational velocity is decreased to $156.2 \mathrm{rad} / \mathrm{s}$; the stable phase difference is increased to $3.72^{\circ}$ from $0.5^{\circ}$, and this is according to theory analysis in Section 5.1 (see Figures 2(b) and 2(c)); the electromagnetic torque of rotor 1 became zero as the power source of induction motor 1 is cut off at $2.5 \mathrm{~s}$ (see (60), $\left.T_{e 2}=0\right)$, and the electromagnetic torque of rotor 1 is increased to $1.4 \mathrm{~N} \cdot \mathrm{m}$ for balancing resistance and frictions of the system.
Figures 3(d) 3(f) describe the responses in $x$-, $y$-, and $\psi$ directions. In the starting stage the displacement in $x$-, $y$-, and $\psi$-axes is by far larger than other stages as the exciting frequency through the resonant region of the vibrating system. During the synchronous state, the displacement in $x$ - and $\psi$-directions is smaller as the stable phase difference is equal to zero. Thus, the exciting forces produced by the two rotors in $x$ - and $\psi$-directions are offset; on the contrary, exciting forces in $y$-direction are additive. In the powersupplying stage, the displacement in $x$-axis and $\psi$-axis is smaller than that in the power-cutting stage because of the increase of the stable phase difference. As the small amplitude of dynamic respond of the oscillating body in $x$-and $\psi$-axis, 


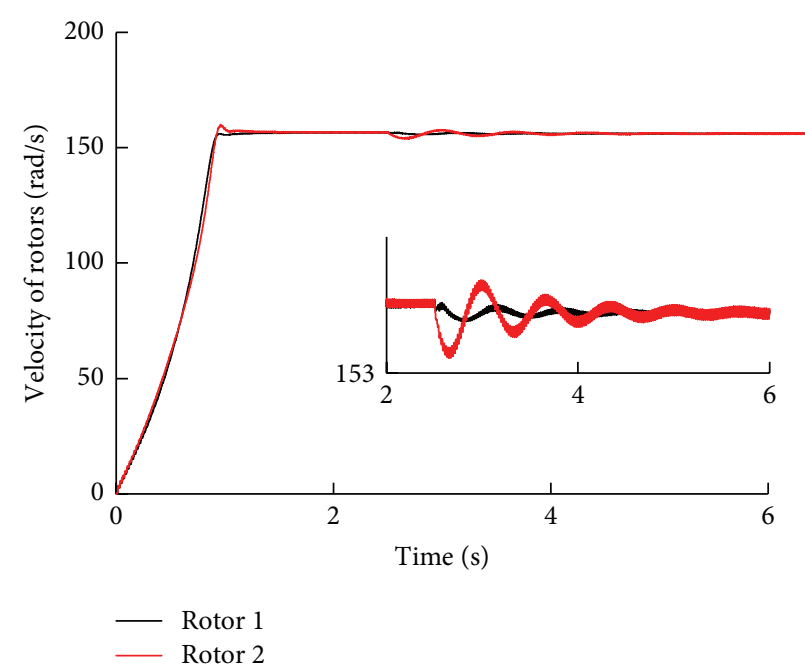

(a)

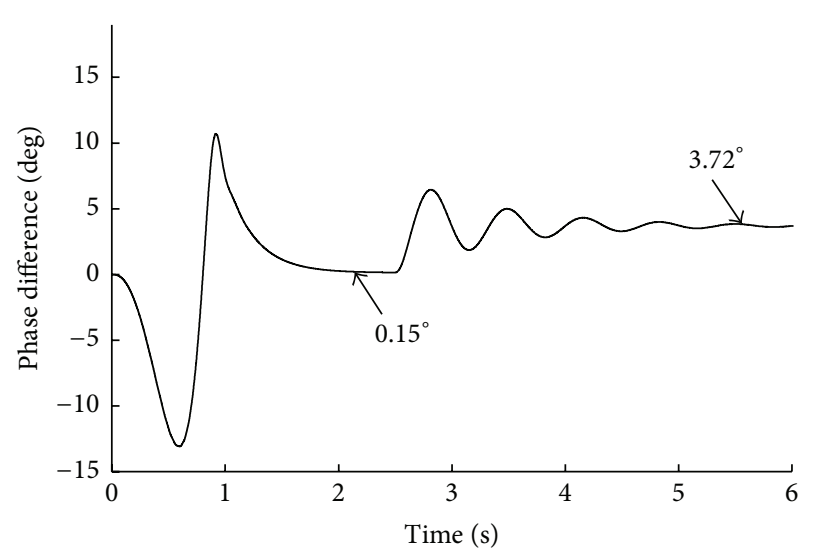

(c)

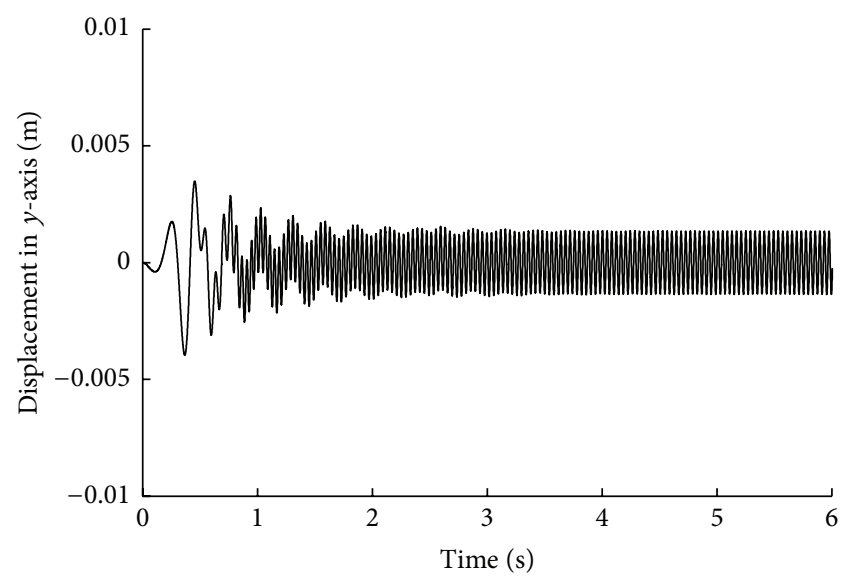

(e)

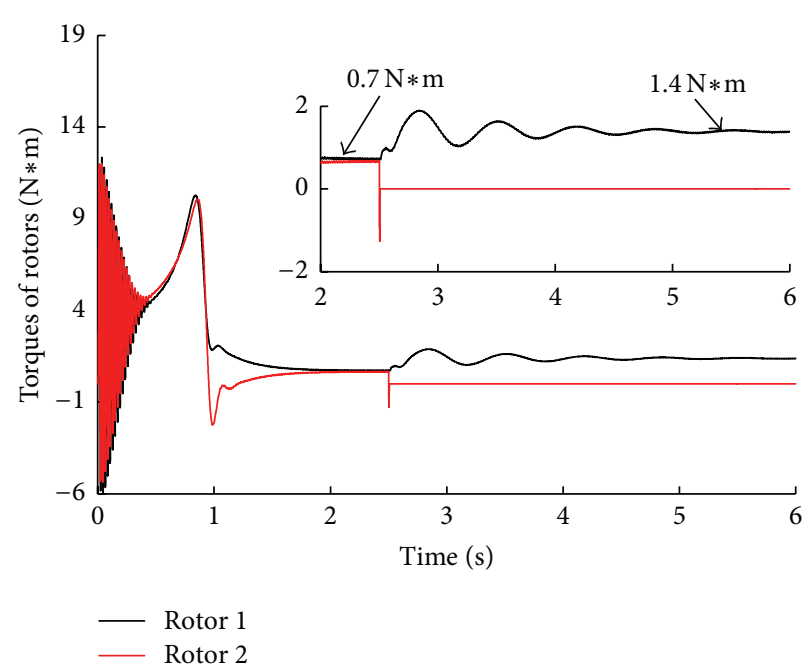

(b)

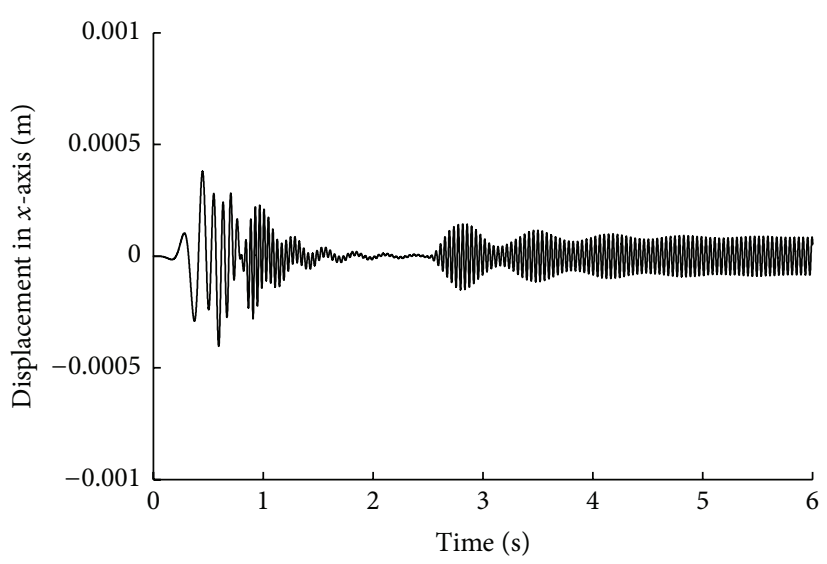

(d)

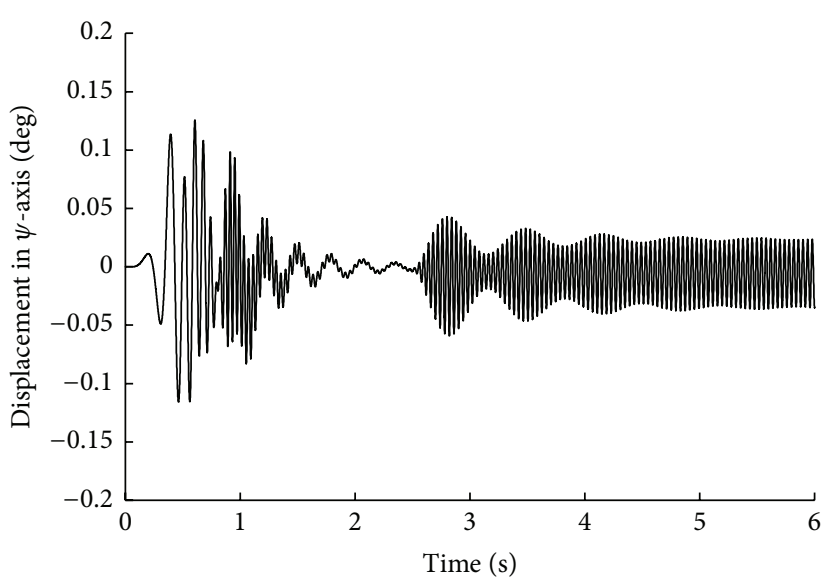

(f)

FIgURE 3: Numerical results for two identical opposite rotors.

in this case, we could consider the oscillating body as a pure linear motion in the $y$-axis.

According to (5) (37), we can deduce the equation of energy balance of the synchronization system.
In the power-supplying stage, the part energy supplied by the two induction motors is dissipated by their friction dampers $\left(W_{1}^{\text {DAMP }}\right.$ and $\left.W_{2}^{\text {DAMP }}\right)$ and the other part is transferred to the oscillating body (synchronization energy $W_{1}^{\text {SYS }}$ 


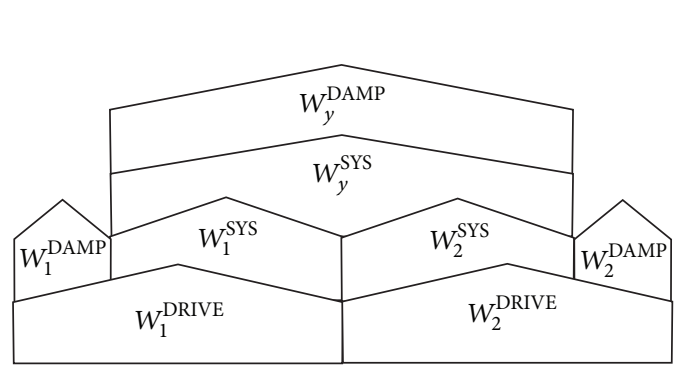

(a) Power-supplying state

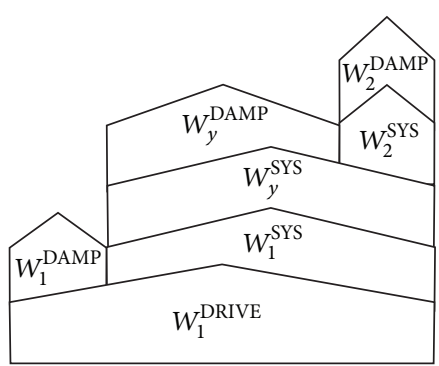

(b) Power-cutting state

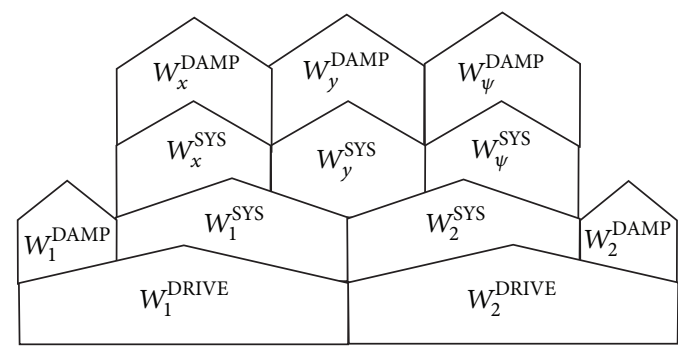

(c) Nonidentical rotors

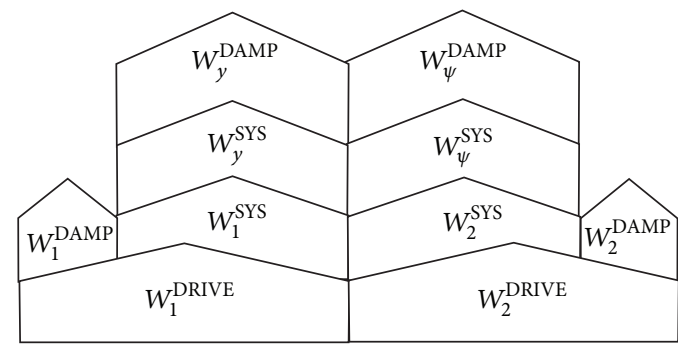

(d) Asymmetric location of rotors

Figure 4: Energy transmission. Figures (a), (b), (c), and (d) are depicted according to (60), (61), (62), and (63), respectively.

and $W_{2}^{\text {SYS }}$ ) when the two rotors rotate synchronously. In addition, the synchronization energy excites the oscillating body $\left(W_{y}^{\text {SYS }}\right)$ and is dissipated by the damper of the oscillating body $W_{y}^{\text {DAMP }}$. Owing to the small values of the energy in $x$ axis and $\psi$-axis (i.e., $W_{x}^{\text {SYS }} \approx 0$ and $W_{\psi}^{\text {SYS }} \approx 0$ ), the energy balance equation of the vibrating system during the powercutting stage can be expressed as

$$
\begin{aligned}
& W_{1}^{\text {DAMP }}+W_{1}^{\text {SYS }}=W_{1}^{\text {DRIVE }}, \\
& W_{2}^{\text {DAMP }}+W_{2}^{\text {SYS }}=W_{2}^{\text {DRIVE }}, \\
& W_{1}^{\text {DAMP }}+W_{1}^{\text {SYS }}+W_{2}^{\text {DAMP }}+W_{2}^{\text {SYS }} \\
& =W_{1}^{\text {DRIVE }}+W_{2}^{\text {DRIVE }}, \\
& W_{1}^{\text {SYS }}+W_{2}^{\text {SYS }}=W_{y}^{\text {SYS }}, \\
& W_{y}^{\text {SYS }}=W_{y}^{\text {DAMP }} .
\end{aligned}
$$

In the power-cutting stage, the power source on the second rotor is cut off, and so the electromagnetic torque of the rotor is equal to zero within the synchronization state; that is, $W_{2}^{\text {DRIVE }}=0$ (see Figure $3(\mathrm{~b})$ ). In this case the synchronization energy $\left(W_{1}^{\text {SYS }}\right.$ ) and dissipated energy $\left(W_{1}^{\text {DAMP }}\right)$ are balanced only by the electromagnetic torque of the first motor through the oscillation of the oscillating body, and synchronization energy $\left(W_{1}^{\mathrm{SYS}}\right)$ is transferred to the oscillating body $\left(W_{y}^{\mathrm{SYS}}\right)$. Then the energy $W_{y}^{\mathrm{SYS}}$ of the oscillating body is balanced by synchronization energy $W_{2}^{\text {SYS }}$ and dissipated energy $\left(W_{y}^{\text {DAMP }}\right)$. At last, synchronization energy $W_{2}^{\text {SYS }}$ is balanced by dissipated energy $\left(W_{2}^{\text {DAMP }}\right)$; thus, maintenance of rotation takes place. Neglecting the energy in $x$ - and $\psi$-directions, the energy balance equation of the system during the power-cutting state can be expressed as

$$
\begin{aligned}
W_{1}^{\mathrm{DAMP}}+W_{1}^{\mathrm{SYS}} & =W_{1}^{\mathrm{DRIVE}}, \\
W_{1}^{\mathrm{SYS}} & =W_{y}^{\mathrm{SYS}}, \\
W_{y}^{\mathrm{SYS}} & =W_{y}^{\mathrm{DAMP}}+W_{2}^{\mathrm{SYS}}, \\
W_{2}^{\mathrm{SYS}} & =W_{2}^{\mathrm{DAMP}} .
\end{aligned}
$$

Figures 4(a)-4(b) illustrate the energy balance of the vibrating system in the power-supplying stage and power-cutting stage, respectively.

5.2.2. Simulation Results for Two Nonidentical Rotors. In calculations of the synchronization ability of the vibrating system excited by the two nonidentical rotors, we consider the following values of the dynamics parameters (nonidentical mass and symmetric location of the two rotors): $r_{l 1}=1.0$, $r_{l}=1.0$, and $\eta=0.6$, and other parameters are also the same as the aforementioned.

Figures 5(a)-5(c) describe rotational velocities, torques, and phase difference of the two nonidentical rotors excited by the identical induction motors, respectively. It can be seen that the synchronous rotational velocity of the rotors stabilizes at vicinity of $156.7 \mathrm{rad} / \mathrm{s}$; the electromagnetic torques of the induction motors are approximately equal to $0.5 \mathrm{~N} \cdot \mathrm{m}$; the phase difference between the two rotors $2 \alpha$ stabilizes at vicinity $-0.3^{\circ}$; this is in accordance with the theoretical analysis above. Figures 5(d) 5(f) describe the responses in $x$ ,$y$-, and $\psi$-directions. In the starting stage the displacement in $x-, y$-, and $\psi$-axes is also by far larger than the stability stage as the exciting frequency through the resonant region 

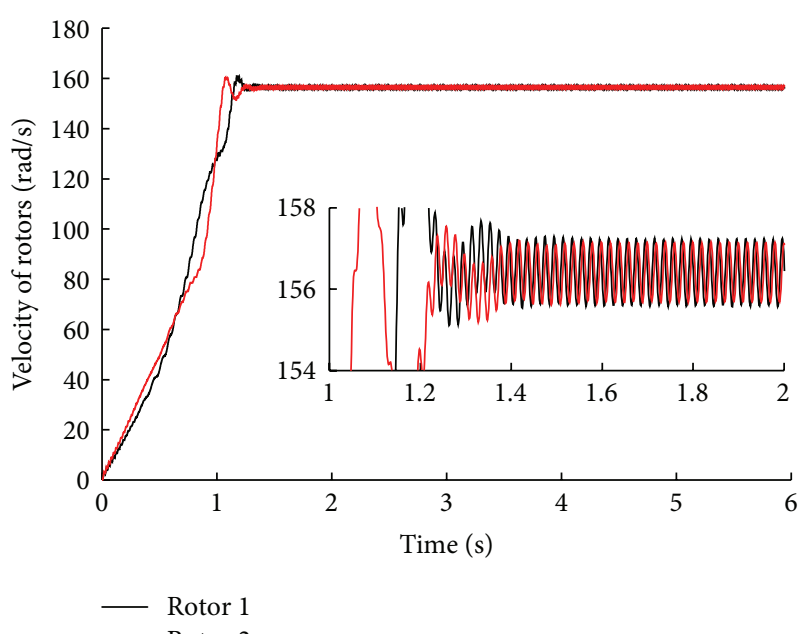

$\begin{array}{ll}- & \text { Rotor } 1 \\ - & \text { Rotor } 2\end{array}$

(a)

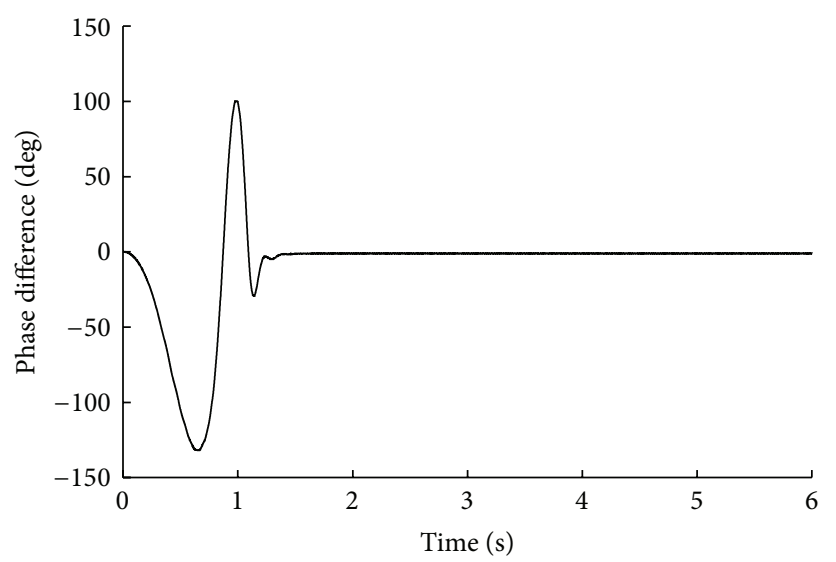

(c)

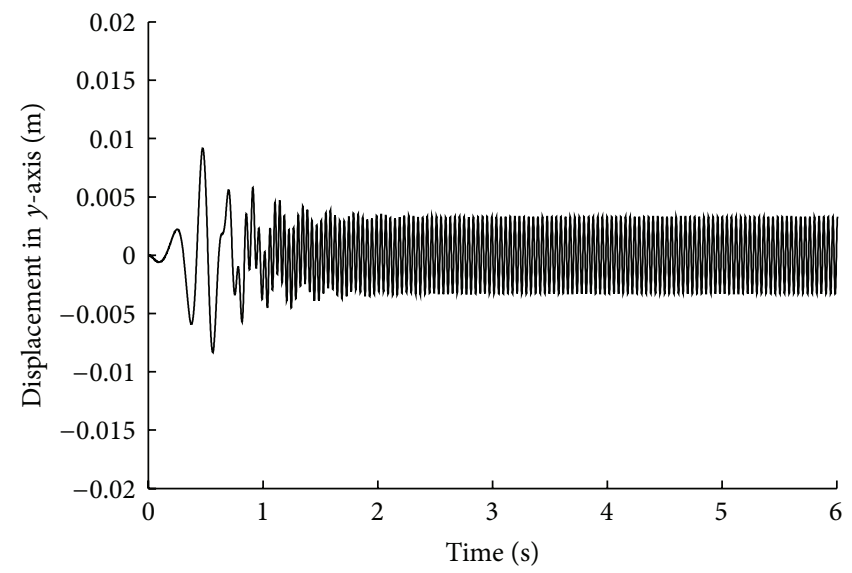

(e)

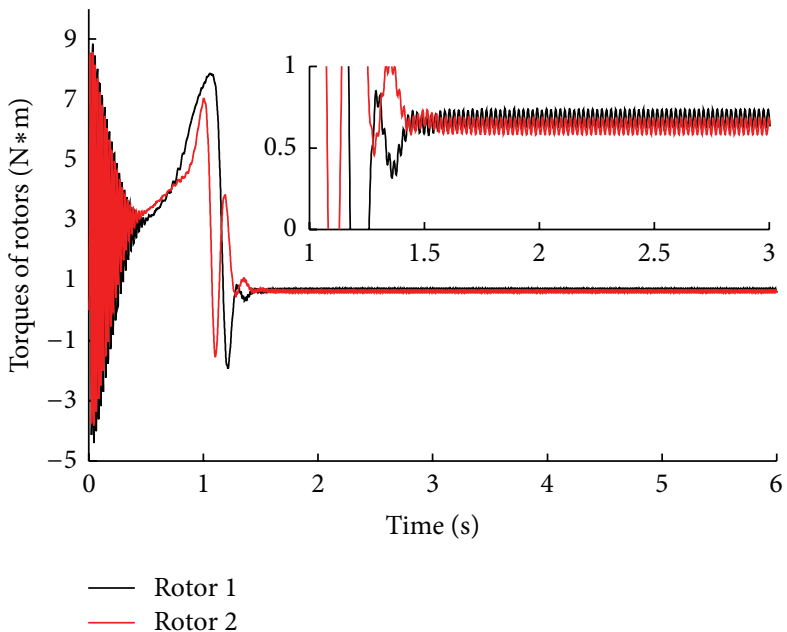

(b)

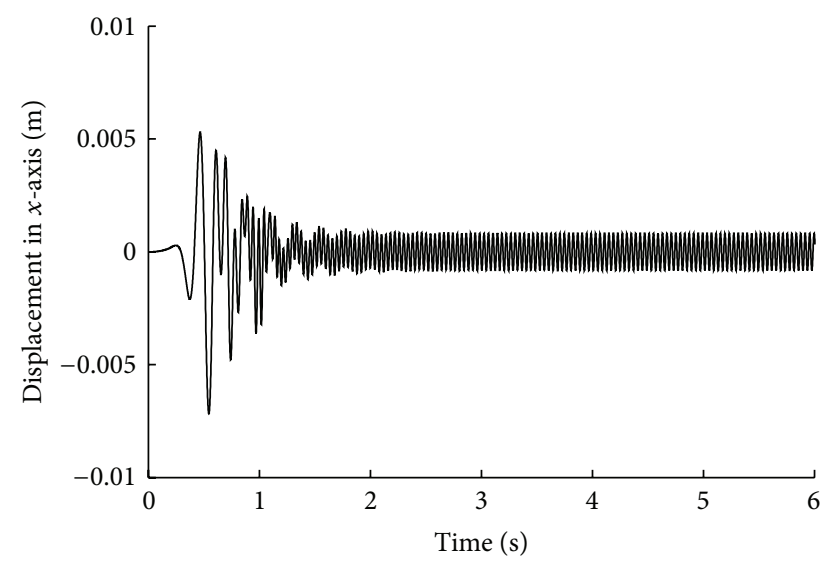

(d)

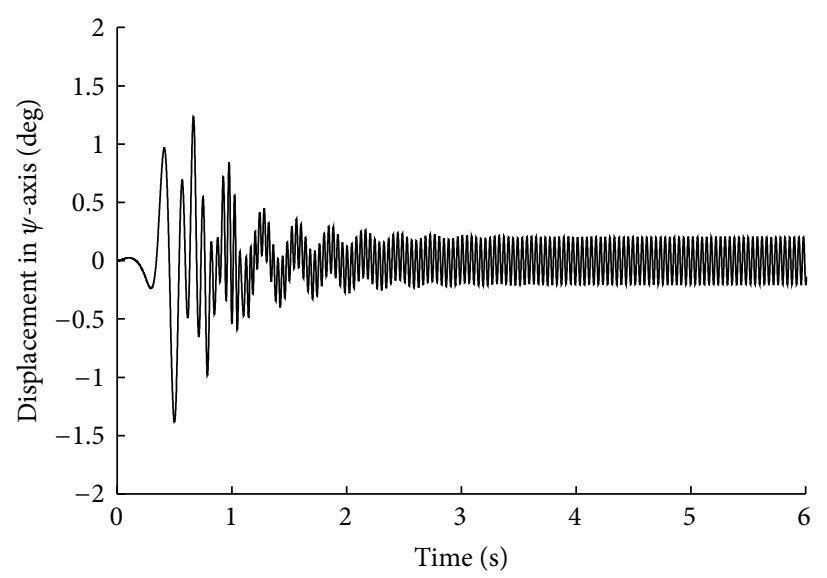

(f)

FIgURE 5: Numerical results for two no-identical opposite rotors.

of the vibrating system. Although the stable phase difference is approximately equal to $-0.3^{\circ}$, the nonidentical mass of the two rotors leads to the larger displacement in $x$-, $y$-, and $\psi$ axes. As a result, the motion type of the oscillating body is an ellipse in $o x y$-plane with a swing in $\psi$-axis.
In the synchronous state, the part energy supplied by the two induction motors is dissipated by their friction dampers $\left(W_{1}^{\text {DAMP }}\right.$ and $\left.W_{2}^{\text {DAMP }}\right)$ and the other part is transferred to the oscillating body (synchronization energy $W_{1}^{\text {SYS }}$ and $W_{2}^{\text {SYS }}$ ). Moreover, the synchronization energy excites the oscillating 
body $\left(W_{x}^{\text {SYS }}, W_{y}^{\text {SYS }}\right.$, and $\left.W_{\psi}^{\text {SYS }}\right)$ and is dissipated by the damper of the oscillating body $\left(W_{x}^{\text {DAMP }}, W_{y}^{\text {DAMP }}\right.$, and $\left.W_{\psi}^{\text {DAMP }}\right)$. So the energy balance equation of the vibrating system during the steady state can be expressed as

$$
\begin{aligned}
& W_{1}^{\text {DAMP }}+W_{1}^{\text {SYS }}=W_{1}^{\text {DRIVE }}, \\
& W_{2}^{\text {DAMP }}+W_{2}^{\text {SYS }}=W_{2}^{\text {DRIVE }}, \\
& W_{1}^{\text {SYS }}+W_{2}^{\text {SYS }}=W_{x}^{\text {SYS }}+W_{y}^{\text {SYS }}+W_{\psi}^{\text {SYS }}, \\
& W_{x}^{\text {SYS }}+W_{y}^{\text {SYS }}+W_{\psi}^{\text {SYS }} \\
& =W_{x}^{\text {DAMP }}+W_{y}^{\text {DAMP }}+W_{\psi}^{\text {DAMP }} .
\end{aligned}
$$

Figure 4(c) shows the energy transmission of the system considering the nonidentical mass of the rotors.

\subsubsection{Simulation Results for Asymmetric Location and Distur-} bance of Rotors. To further verify the synchronous stability of the two rotors, it is necessary to perform simulations for the vibrating system with a phase disturbance on the rotors. We consider the following values of the system parameters (identical mass and asymmetric location of the two rotors): $r_{l 1}=0.5, r_{l}=3.0$, and $\eta=1$, and a disturbance of phase $\pi / 2$ is added to the second rotor at $3 \mathrm{~s}$. Other parameters of the vibrating system are also the same as the aforementioned.

Figures 6(a)-6(c) show rotational velocities, torques, and phase difference of the two rotors installed asymmetrically, respectively. Without the disturbance, the synchronous rotational velocity of the rotors stabilizes at vicinity of $156.4 \mathrm{rad} / \mathrm{s}$; the electromagnetic torque of the induction motors is approximately equal to $0.5 \mathrm{~N} \cdot \mathrm{m}$; the stable phase difference between the two rotors $2 \alpha$ is equal to $0.5^{\circ}$. With the disturbance, the electromagnetic torques where the coupling torques act on the second rotors become the load torques. Oppositely, the load torques on the first rotor become the driving torques (see Figure 6(b)). This phenomenon leads to the decrease of the rotational velocity of the first rotor and the increase of that of the second rotor (see Figure 6(a)). With the self-adjustment of the electromagnetic torque, the disturbed vibrating system gradually returns to the previous synchronization state. In addition, with the above process of disturbance added, the displacements of the oscillating body have a large value as the phase difference changes. If a disturbance of $\pi / 2$ phase is added to the first rotor, the disturbed vibrating system could also return to the previous synchronization state. The simulation results demonstrate the stability of synchronization of the system.

Figures 6(d)-6(f) describe the responses of the system in $x$-, $y$-, and $\psi$-directions. In the synchronous state the amplitude of the oscillating body can be ignored as the counteraction of the vibration force produced by two identical rotors in $x$-direction; on the contrary, the amplitude of the oscillating body in $y$-direction is by far larger than that in $x$-direction on the account of the addition of the vibration force of the two rotors. Owing to the asymmetric location of the two induction motors, the oscillating body also swings with a larger value in $\psi$-direction and so the motion type of the oscillating body is linear in $y$-direction with a swing in $\psi$-direction.

In the synchronous state, the part energy supplied by the two induction motors is dissipated by their friction dampers $\left(W_{1}^{\text {DAMP }}\right.$ and $W_{2}^{\text {DAMP }}$ ) and the other part is transferred to the oscillating body (synchronization energy $W_{1}^{\text {SYS }}$ and $W_{2}^{\text {SYS }}$ ). Moreover, the synchronization energy excites the oscillating body $\left(W_{y}^{\text {SYS }}, W_{\psi}^{\text {SYS }}\right)$ and is dissipated by the damper of the oscillating body $\left(W_{y}^{\text {DAMP }}, W_{\psi}^{\text {DAMP }}\right)$. Owing to the small values of the energy in $x$-direction (i.e., $W_{x}^{\text {SYS }} \approx 0, W_{x}^{\text {DAMP }} \approx 0$ ), the energy balance equation of the vibrating system during the synchronous state can be expressed as

$$
\begin{aligned}
W_{1}^{\mathrm{DAMP}}+W_{1}^{\mathrm{SYS}} & =W_{1}^{\mathrm{DRIVE}}, \\
W_{2}^{\mathrm{DAMP}}+W_{2}^{\mathrm{SYS}} & =W_{2}^{\mathrm{DRIVE}}, \\
W_{1}^{\mathrm{SYS}}+W_{2}^{\mathrm{SYS}} & =W_{y}^{\mathrm{SYS}}+W_{\psi}^{\mathrm{SYS}}, \\
W_{y}^{\mathrm{SYS}}+W_{\psi}^{\mathrm{SYS}} & =W_{y}^{\mathrm{DAMP}}+W_{\psi}^{\mathrm{DAMP}} .
\end{aligned}
$$

Figure $4(\mathrm{~d})$ shows the energy transmission of the asymmetric location of the two rotors when the two rotors rotate synchronously.

\section{Conclusions}

In summary, the energy balance method is employed to extend investigation of a generalization of the vibrating system on synchronization and synchronous stability of unbalanced rotors excited by induction motors with fast rotation, on which we show how the energy is transferred between rotors via the oscillating body allowing the implementation of synchronization of rotors. In order to ensure the synchronous and stable operation of the rotors, the dynamics parameters should satisfy both the condition of synchronization and the criterion of synchronous stability. To prove the correctness of the theoretical analysis, many features of the vibrating system are computed and discussed with computer simulations. It can be found that, in the power-supplying state no matter how larger value of parameters $r_{l}$ and $\eta$ is, the phase difference between the two rotors can stabilize at vicinity of zero degree on account of the offsetting of the electromagnetic moments of the rotors. However, the value of the stable phase difference in the power-cutting state is larger than the power-supplying state since the electromagnetic moments of the rotor maintain the synchronous operation of the system. While the vibrating system is disturbed by external forces, the rotors can also return to its previous synchronous state. The proposed method may be useful for analyzing another similar synchronization and stability of unbalanced rotors excited by induction motors in vibrating systems. 


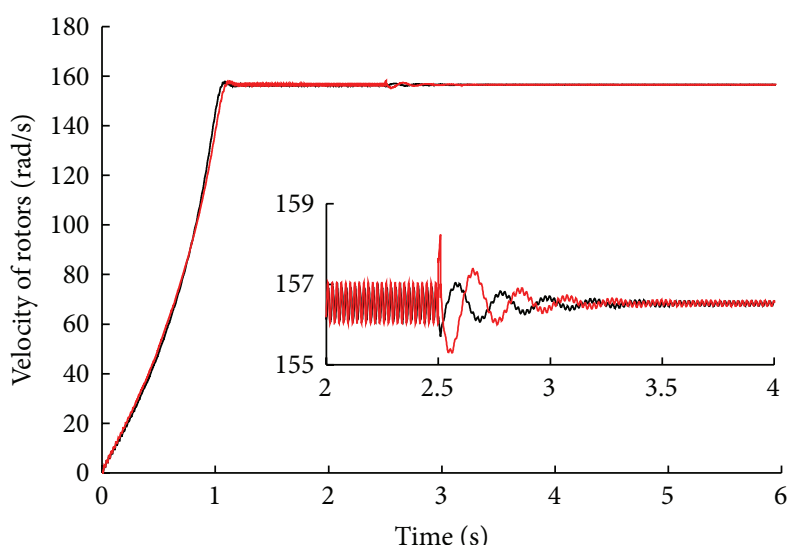

- Rotor 1

- Rotor 2

(a)

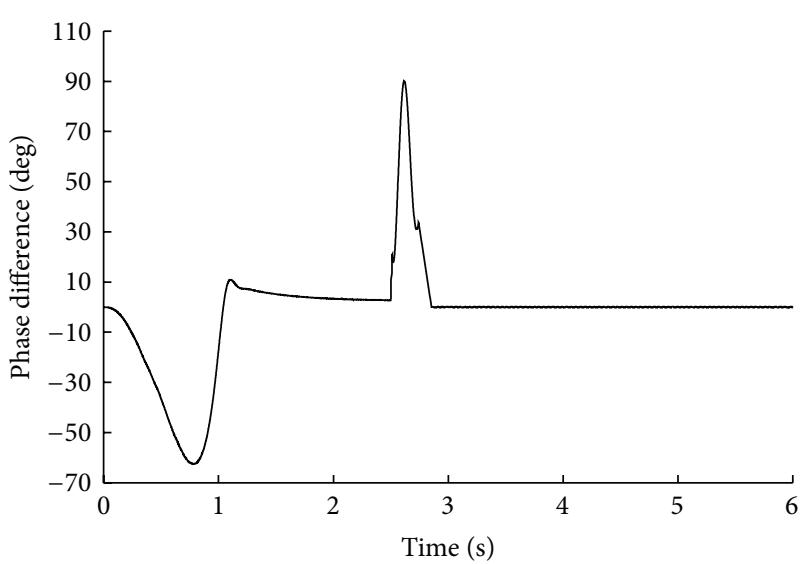

(c)

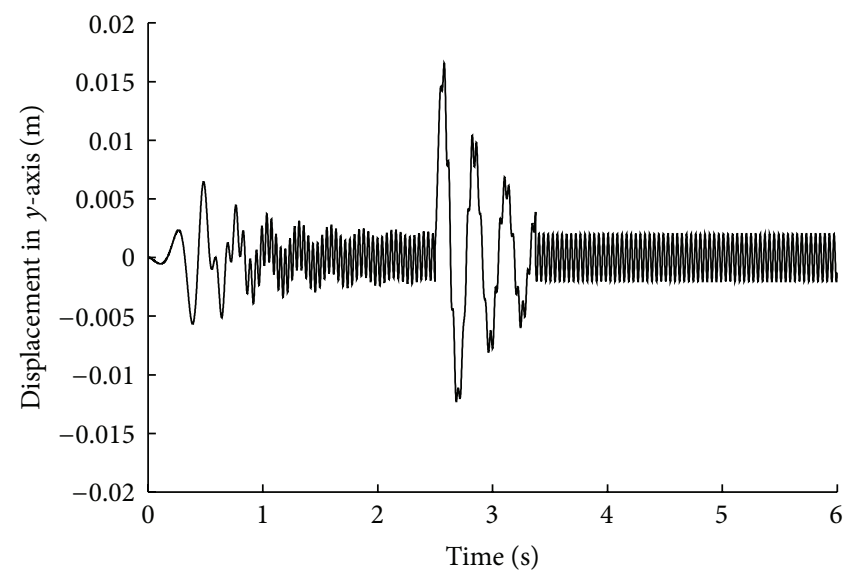

(e)

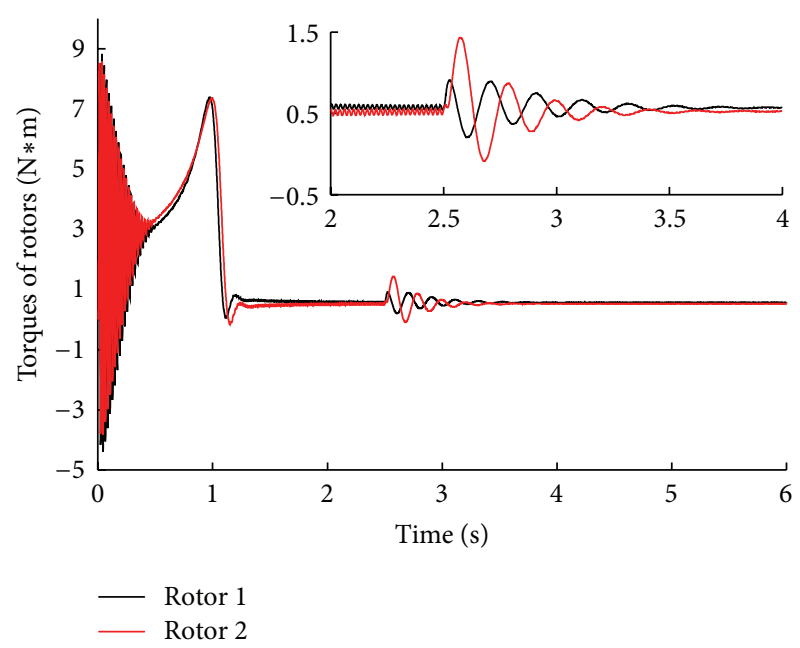

(b)

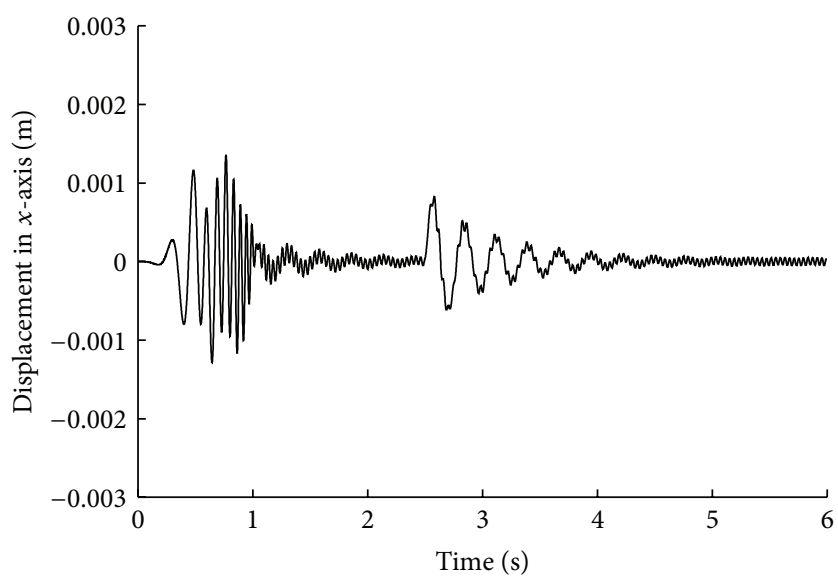

(d)

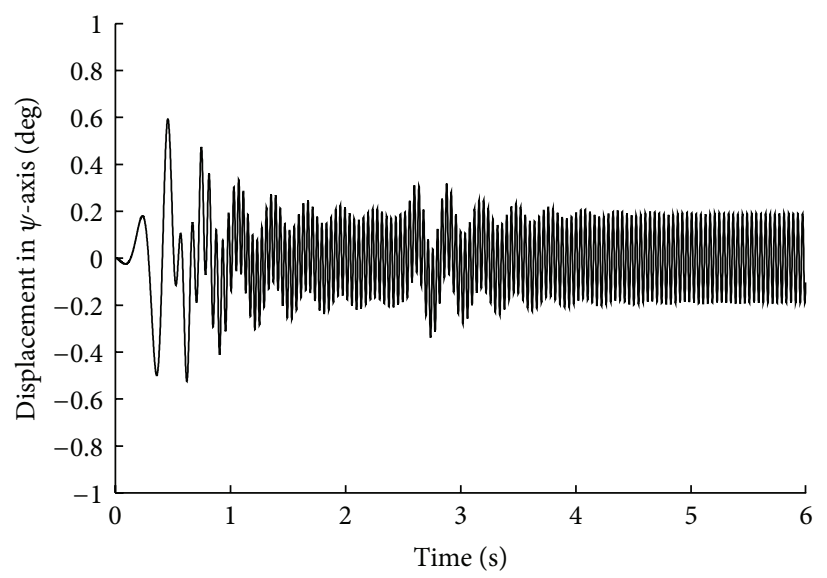

(f)

FIGURE 6: Numerical results for the asymmetric location and disturbance of rotors. 


\section{Conflict of Interests}

The authors declare that there is no conflict of interests regarding the publication of this paper.

\section{Acknowledgment}

This study is supported by National Natural Science Foundation of China (Grant no. 51074132).

\section{References}

[1] C. Huygens, Horologium Pscilatorium, F. Muguet, Paris, France, 1673.

[2] J. Rayleigh, Theory of Sound, Dover Publications, New York, NY, USA, 1945.

[3] B. van der Pol, "Theory of amplitude of free and forced triode vibration," Radio Review, vol. 1, pp. 701-710, 1920.

[4] I. I. Blekhman, Synchronization in Science and Technology, ASME Press, New York, NY, USA, 1988.

[5] B. C. Wen, J. Fan, C. Y. Zhao, and W. L. Xiong, Vibratory Synchronization and Controlled Synchronization in Engineering, Science Press, Beijing, China, 2009.

[6] X. Zhang, B. Wen, and C. Zhao, "Vibratory synchronization transmission of two exciters in a super-resonant vibrating system," Journal of Mechanical Science and Technology, vol. 28, no. 6, pp. 2049-2058, 2014.

[7] X. Zhang, B. Wen, and C. Zhao, "Vibratory synchronization and coupling dynamic characteristics of multiple unbalanced rotors on a mass-spring rigid base," International Journal of Non-Linear Mechanics, vol. 60, pp. 1-8, 2014.

[8] P. Fang, Q. Yang, Y. Hou, and Y. Chen, "Theoretical study on self-synchronization of two homodromy rotors coupled with a pendulum rod in a far-resonant vibrating system," Journal of Vibroengineering, vol. 16, pp. 2188-2204, 2014.

[9] L. Sperling, B. Ryzhik, C. Linz, and H. Duckstein, "Simulation of two-plane automatic balancing of a rigid rotor," in Proceedings of the 2nd International Conference on Control of Oscillations and Chaos (COC '00), pp. 351-365, July 2000, Elsevier, St. Petersburg, Russia, 2002.

[10] J. M. Balthazar, J. L. P. Felix, and R. M. L. R. F. Brasil, "Short comments on self-synchronization of two non-ideal sources supported by a flexible portal frame structure," Journal of Vibration and Control, vol. 10, no. 12, pp. 1739-1748, 2004.

[11] J. M. Balthazar, J. L. P. Felix, and R. M. Brasil, "Some comments on the numerical simulation of self-synchronization of four non-ideal exciters," Applied Mathematics and Computation, vol. 164, no. 2, pp. 615-625, 2005.

[12] V. Jovanovic and S. Koshkin, "Synchronization of Huygens' clocks and the Poincaré method," Journal of Sound and Vibration, vol. 331, no. 12, pp. 2887-2900, 2012.

[13] P. Koluda, P. Perlikowski, K. Czolczynski, and T. Kapitaniak, "Synchronization of two self-excited double pendula," The European Physical Journal: Special Topics, vol. 223, no. 4, pp. 613-629, 2014.

[14] P. Koluda, P. Perlikowski, K. Czolczynski, and T. Kapitaniak, "Synchronization configurations of two coupled double pendula," Communications in Nonlinear Science and Numerical Simulation, vol. 19, no. 4, pp. 977-990, 2014.

[15] P. Koluda, P. Brzeski, and P. Perlikowski, "Dynamics of $\mathrm{n}$ coupled double pendula suspended to the moving beam,"
International Journal of Structural Stability and Dynamics, vol. 14, no. 8, Article ID 1440028, 2014.

[16] M. Kapitaniak, K. Czolczynski, P. Perlikowski, A. Stefanski, and T. Kapitaniak, "Synchronous states of slowly rotating pendula," Physics Reports, vol. 541, no. 1, pp. 1-44, 2014.

[17] M. Kapitaniak, M. Lazarek, M. Nielaczny, K. Czolczynski, P. Perlikowski, and T. Kapitaniak, "Synchronization extends the life time of the desired behavior of globally coupled systems," Scientific Reports, vol. 4, article 4391, 2014.

[18] J. Pena-Ramírez, R. H. B. Fey, and H. Nijmeijer, "In-phase and anti-phase synchronization of oscillators with Huygens' coupling," Cybernetics and Physics, vol. 1, pp. 58-66, 2012.

[19] J. P. N. Ramírez, A. A. Aguirre, R. H. B. Fey, and H. Nijmeijer, "Effects of time delay in the synchronized motion of oscillators with Huygens' coupling," in Proceedings of the 3rd IFAC CHAOS Conference, Cancún, Mexico, June 2012.

[20] D. Yurchenko, A. Naess, and P. Alevras, "Pendulum's rotational motion governed by a stochastic Mathieu equation," Probabilistic Engineering Mechanics, vol. 31, pp. 12-18, 2013. 


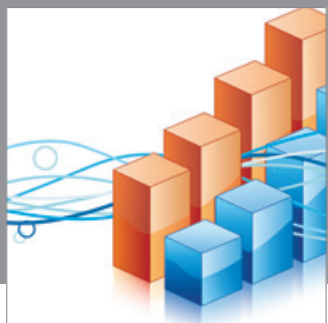

Advances in

Operations Research

mansans

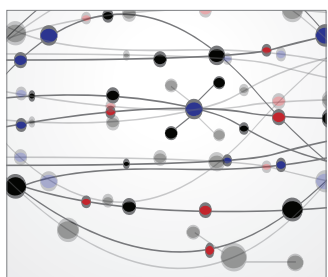

The Scientific World Journal
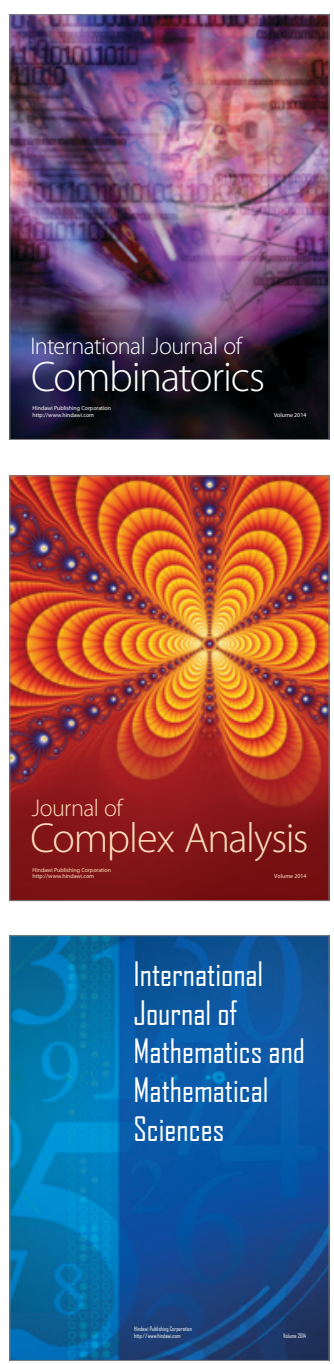
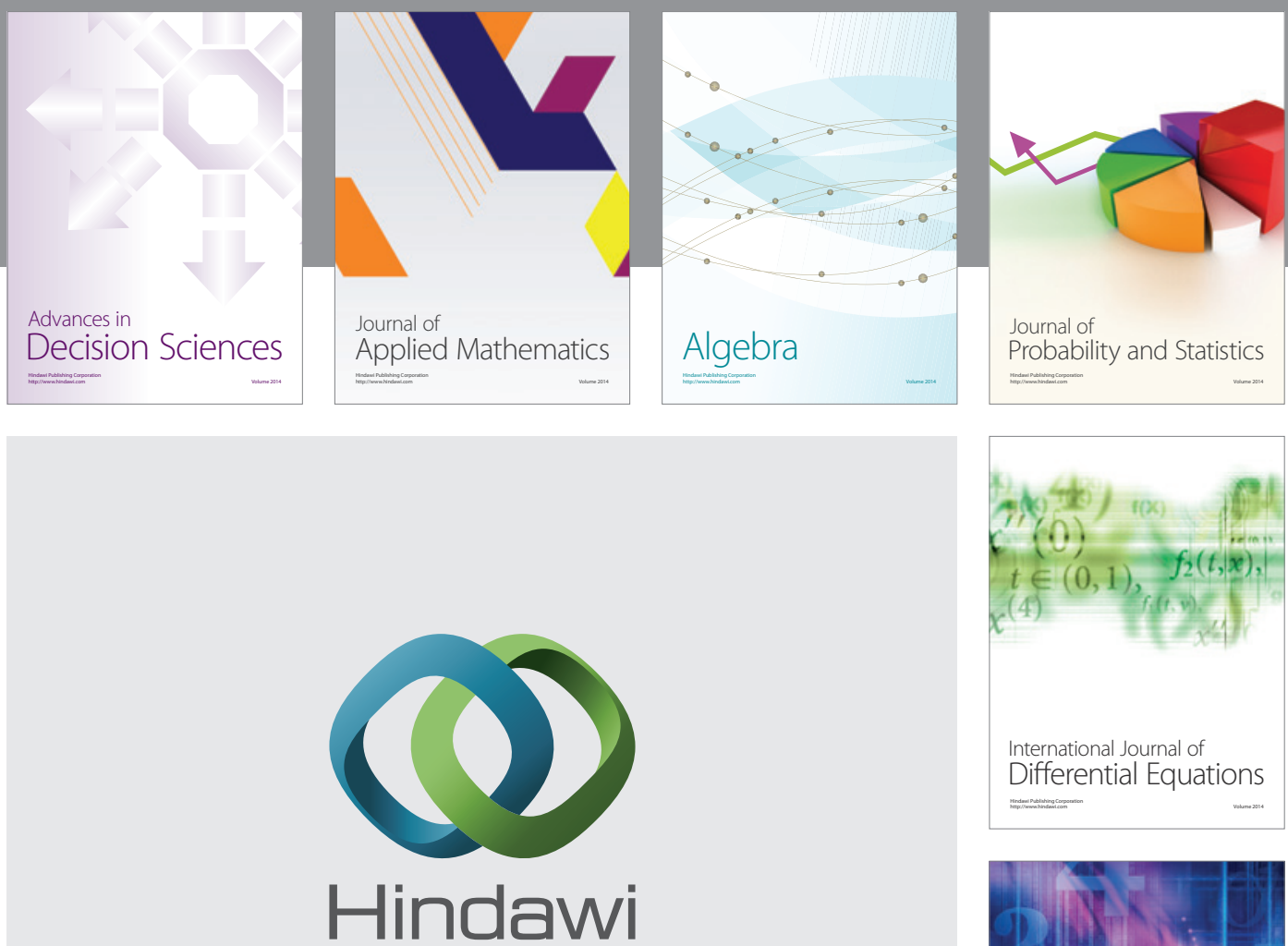

Submit your manuscripts at http://www.hindawi.com
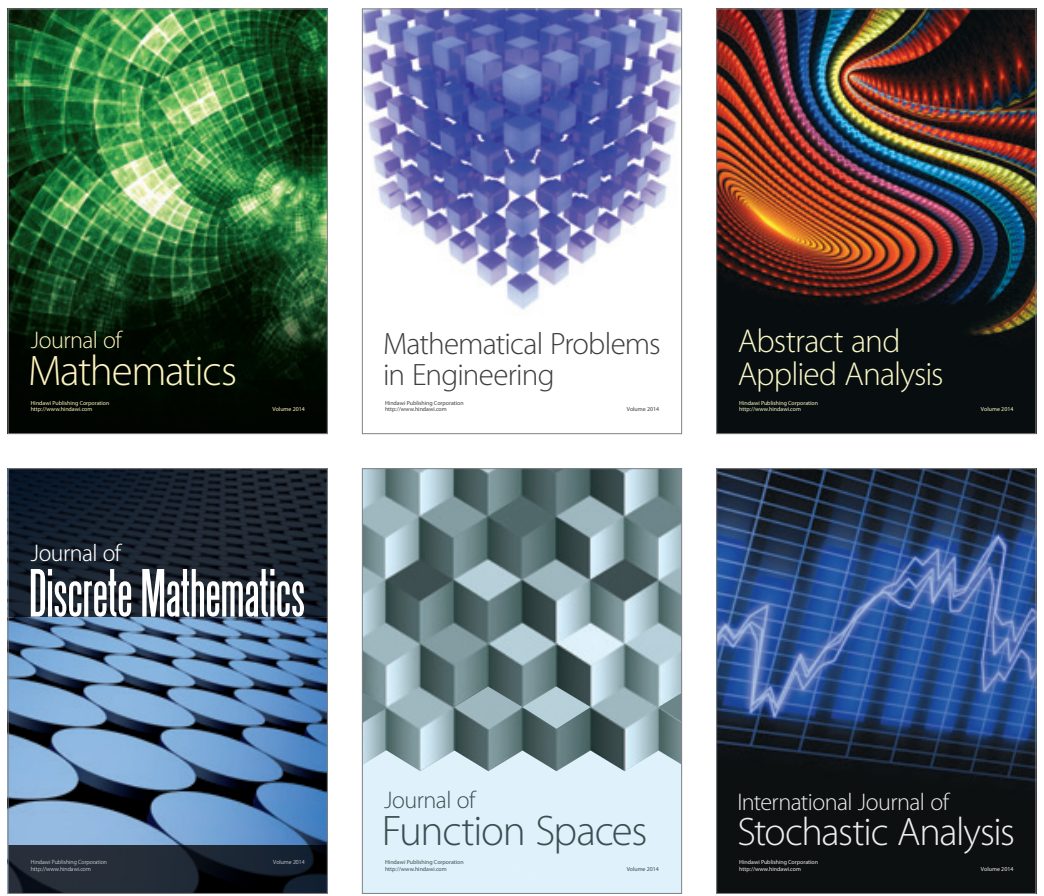

Journal of

Function Spaces

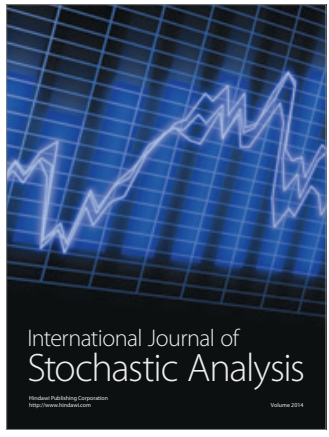

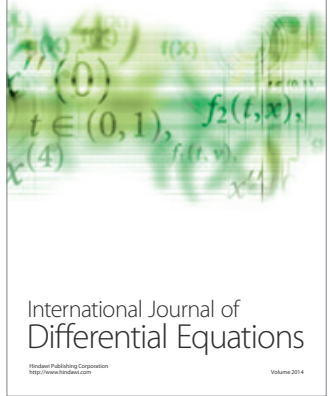
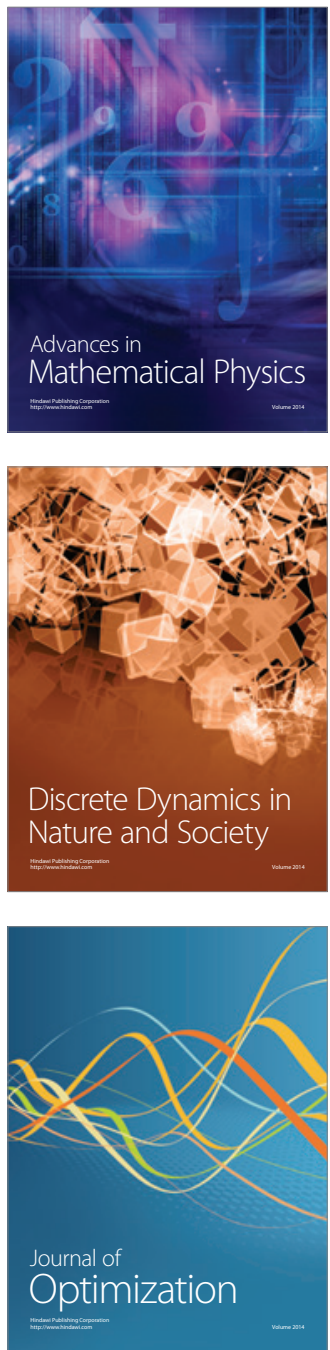